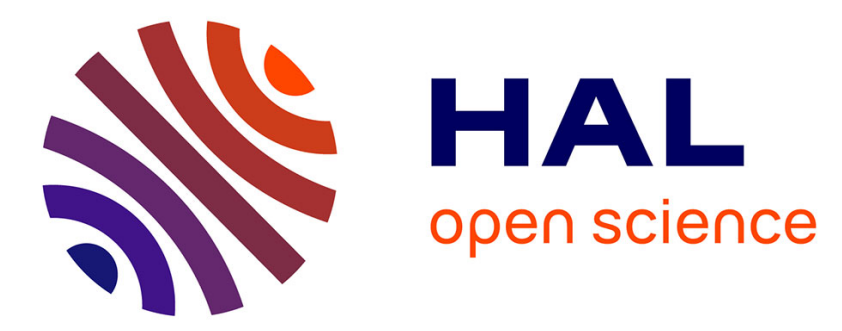

\title{
Model updating in structural dynamics - Uncertainties on the position and orientation of sensors and actuators
}

Anas Batou

\section{To cite this version:}

Anas Batou. Model updating in structural dynamics - Uncertainties on the position and orientation of sensors and actuators. Journal of Sound and Vibration, 2015, 10.1016/j.jsv.2015.06.017 . hal01162717

\section{HAL Id: hal-01162717 https://hal.science/hal-01162717}

Submitted on 18 Jul 2015

HAL is a multi-disciplinary open access archive for the deposit and dissemination of scientific research documents, whether they are published or not. The documents may come from teaching and research institutions in France or abroad, or from public or private research centers.
L'archive ouverte pluridisciplinaire HAL, est destinée au dépôt et à la diffusion de documents scientifiques de niveau recherche, publiés ou non, émanant des établissements d'enseignement et de recherche français ou étrangers, des laboratoires publics ou privés. 


\title{
Model updating in structural dynamics - Uncertainties on the position and orientation of sensors and actuators
}

\author{
A. Batou \\ Université Paris-Est, Laboratoire Modélisation et Simulation Multi Echelle, MSME UMR \\ 8208 CNRS, 5 bd Descartes, 77454 Marne-la-Vallee, France \\ E-mail: anas.batou@univ-paris-est.fr, \\ Tel.: +33160957792, fax: +33160857799
}

\begin{abstract}
This research concerns the updating of computational models in presence of uncertainties related to the position and the orientation of the sensors and actuators. Such uncertainties yield uncertainties in the correspondence between the experimental dynamical responses and the dynamical responses calculated using the computational model. These uncertainties in the response increase with frequency and have to be taken into account when updating the parameters of the computational model in order to obtain a robust estimation of these parameters. This paper provides a complete methodology to take into account and analyse such uncertainties. Furthermore, an optimal sensor placement method is proposed so that (1) the measured data are as sensitive as possible with respect to updating parameters and (2) the measured data are as robust as possible with respect to position/orientation uncertainties. The methodologies developed here are illustrated through two numerical applications.
\end{abstract}

Keywords: model updating, Bayesian inference, uncertain position, optimal sensor placement

\section{Introduction}

In the context of computational structural dynamics, the model updating methods have received much interest during the last four decades [25, 10]. The researches related to model updating methods have been focused in two main classes of methods. For the first class of methods referred as global 
methods [1, 11, 21, 29], the assembled stiffness and mass matrices are directly modified regardless the parameters of the computational model. On the contrary, for the local methods, some chosen physical parameters of the computational model are updated (see for instance $[4,7]$ ). The research presented in this paper is related to local updating methods for which the optimal values of the updating parameters are chosen as the ones that make the experimental observations as closed as possible to the corresponding observations calculated using the computational model. In the Bayesian updating method [9, 2], the updating parameters are replaced by random variables for which the prior probability distribution has to be chosen and an output prediction error is introduced in order to take into account modelling errors and measurement noise. Then a posterior probability distribution can be calculated for the updating parameters using the Bayes rule.

In structural dynamics, the experimental observations are, in general, constructed using experimental measurements that are collected using actuators (hammers, shakers, ...) and sensors (accelerometers, deformation gauges, piezoelectric sensors, ...). The location and orientation of the sensors/actuators on the real structures have to be known exactly so that the experimental data corresponds to the data calculated using the computational model. Unfortunately, when dealing with real on-site structures, many sources of uncertainties may deteriorate the correspondence between the experimental locations/orientations and the computational locations/orientations: (1) there may be some slight differences between the real geometry of the measured structure and the geometry of the computational model, (2) there may be some manipulation errors during the placement of the sensors/actuators (more especially for positions which are hard to access), (3) some information may have not been addressed correctly by the experimenters to the engineers, and so on. The presence of such uncertainties induces uncertainties on the outputs of the computational model and as a consequence induces uncertainties in the updated values of the parameters of the computational model.

There are three objectives in this paper. The first objective consists in constructing a probabilistic model of uncertainties related to the position and orientation of the sensors and actuators. This probabilistic model has to be adapted to the framework of a Finite Element (FE) analysis in order to obtain a stochastic computational model that will be used to propagate these uncertainties into the computational dynamical response. The second objective of this paper is to provide a robust model updating methodology in presence of such uncertainties. The methodology proposed here is based 
on a Bayesian approach. The third objective of this paper is to provide a methodology for an optimal placement of the sensors/actuators so that the measurements yields maximum information for the updating procedure which means that the measured data (1) should be sensitive to the updating parameter and (2) should be robust with respect to the position/orientation uncertainties. The method proposed here is based on Information theory [22, $12,13,16,17]$ and more precisely the use of the Kullback-Leibler divergence to measure the gain of information provided by the measurement configuration.

Section 2 is devoted to the construction of the nominal computational model. Then in Section 3, the probabilistic model of position/orientation uncertainties is constructed. Section 4 concerns the model updating in presence of positioning uncertainties and Section 5 is devoted to the optimal sensor/actuator placement. Finally two numerical applications are analysed in Section 6 in order to illustrate and verify the methodology.

\section{Nominal computational model}

In this section, the nominal computational model representing a real instrumented structure is described. We are interested in the frequency response of a linear damped structure, occupying a domain $\Omega$, in the frequency band of analysis $B=\left[\omega_{\min }, \omega_{\max }\right]$. Let $\boldsymbol{x}$ be the coordinates of a point in $\Omega$. Let $\boldsymbol{u}(\boldsymbol{x}, \omega)$ be the displacement field defined in the frequency domain with values in $\mathbb{C}^{3}$. The nominal computational model is constructed using the FE method. It is assumed that there are no rigid body modes. Let $m$ be the total number of degrees-of-freedom in the FE model. For all $\omega \in B$, the vector $\mathbb{U}(\omega) \in \mathbb{R}^{m}$ of the $m$ degrees-of-freedom is the solution of the following matrix equation

$$
\left(-\omega^{2}[\mathbb{M}]+i \omega[\mathbb{D}]+[\mathbb{K}]\right) \mathbb{U}(\omega)=\mathbb{F}(\omega),
$$

in which $[\mathbb{M}],[\mathbb{D}]$ and $[\mathbb{K}]$ are the $(m \times m)$ mass, damping and stiffness matrices and where $\mathbb{F}(\omega)$ is the vector of the external forces. The reduced nominal computational model is constructed using the modal analysis method [15]. The $n$ first eigenvalues $0<\lambda_{1} \leq \lambda_{2} \leq \ldots \leq \lambda_{n}$ associated with the elastic modes $\left\{\phi_{1}, \phi_{2}, \ldots, \phi_{n}\right\}$ are solutions of the following generalized eigenvalue problem

$$
[\mathbb{K}] \phi=\lambda[\mathbb{M}] \phi
$$


The reduced-order nominal computation model is obtained by projecting the nominal computation model on the subspace spanned by the $n$ first elastic modes calculated using Eq. (2). Let $[\Phi]$ be the $m \times n$ matrix whose columns are the $n$ first elastic modes. We then introduce the approximation $\mathbb{U}^{(n)}(\omega)$ of $\mathbb{U}(\omega)$ such that

$$
\mathbb{U}(\omega) \simeq \mathbb{U}^{(n)}(\omega)=[\Phi] \boldsymbol{q}(\omega),
$$

in which the vector $\boldsymbol{q}(\omega)$ is the vector of the $n$ generalized coordinates and is the solution of the following reduced matrix equation

$$
\left(-\omega^{2}[\widetilde{M}]+i \omega[\widetilde{D}]+[\widetilde{K}]\right) \boldsymbol{q}(\omega)=\tilde{\boldsymbol{f}}(\omega),
$$

in which $[\widetilde{M}]=[\Phi]^{T}[\mathbb{M}][\Phi],[\widetilde{D}]=[\Phi]^{T}[\mathbb{D}][\Phi]$ and $[\widetilde{K}]=[\Phi]^{T}[\mathbb{K}][\Phi]$ are the $n \times n$ mass, damping and stiffness generalized matrices, and where $\widetilde{\boldsymbol{f}}(\omega)=$ $[\Phi]^{T} \mathbb{F}(\omega) \in \mathbb{R}^{n}$ is the vector of the generalized forces.

The nominal experimental configuration of the structure is equipped with $n_{s}$ sensors and $n_{a}$ actuators. For each sensor $s_{i}, i=1, \ldots, n_{s}$ the acceleration is measured following the three directions $\boldsymbol{e}_{x}, \boldsymbol{e}_{y}$ and $\boldsymbol{e}_{z}$. For each actuator $a_{i}$, $i=1, \ldots, n_{a}$, a three dimensional point force is applied. It is assumed that the nominal locations of the sensors and actuators correspond to nodes of the mesh of the FE model. Let $n_{\mathrm{obs}}=3 n_{s}$ and $n_{\mathrm{exc}}=3 n_{a}$. Let $\mathbb{U}^{\mathrm{sens}, \mathrm{i}}(\omega)$ in $\mathbb{C}^{3}$ denote the three translation DOFs observed by sensor $s_{i}$ and let $\mathbb{U}^{\mathrm{sens}}(\omega)=$ $\left(\mathbb{U}^{\text {sens }, 1}(\omega), \ldots, \mathbb{U}^{\text {sens, } n_{\mathrm{s}}}(\omega)\right)$ in $\mathbb{C}^{n_{\text {obs }}}$ be the observed displacement vector. Let $\mathbb{F}^{\text {act,i }}(\omega)$ in $\mathbb{C}^{3}$ denote the point force applied by actuator $a_{i}$ and let $\mathbb{F}^{\text {act }}(\omega)=$ $\left(\mathbb{F}^{\text {act, } 1}(\omega), \ldots, \mathbb{F}^{\text {act, } \mathrm{n}_{\mathrm{a}}}(\omega)\right)$ in $\mathbb{C}^{n_{\mathrm{exc}}}$ be the actuator force vector. The observed displacement vector is linked to the generalized coordinates vector by the relation

$$
\mathbb{U}^{\text {sens }}(\omega)=[O] \mathbb{U}^{(n)}(\omega)=[O][\Phi] \boldsymbol{q}(\omega),
$$

and the actuator force vector is linked to the generalized forces vector by the relation

$$
\widetilde{\boldsymbol{f}}(\omega)=[\Phi]^{T} \mathbb{F}(\omega)=[\Phi]^{T}[C] \mathbb{F}^{\text {act }}(\omega) .
$$

In Eqs. (5) and (6), $[O]$ and $[C]$ are two $\left(n_{\mathrm{obs}} \times m\right)$ localization matrices which have elements equal to zero or one.

Then, using Eqs. (4), (5) and (6), for all $\omega$ in $B$, the observed displacement vector $\mathbb{U}^{\text {sens }}(\omega)$ is written as

$$
\mathbb{U}^{\text {sens }}(\omega)=[O][H(\omega)][C] \mathbb{F}^{\text {act }}(\omega),
$$


in which the frequency response function $[H(\omega)]$ with values in the $(m \times m)$ complex matrices is such that

$$
[H(\omega)]=[\Phi]\left(-\omega^{2}[\widetilde{M}]+i \omega[\widetilde{D}]+[\widetilde{K}]\right)^{-1}[\Phi]^{T}
$$

The response is calculated at $n_{\text {freq }}$ frequencies $\omega_{1}, \ldots, \omega_{n_{\text {freq }}}$ belonging to frequency band $B$. We then introduce the output vector $\boldsymbol{z}$ in $\mathbb{R}^{n_{z}}$ such that

$$
\boldsymbol{z}=\hat{\boldsymbol{z}}\left(\mathbb{U}^{\text {sens }}\left(\omega_{1}\right), \ldots, \mathbb{U}^{\text {sens }}\left(\omega_{n_{\text {freq }}}\right)\right)
$$

where $\hat{\boldsymbol{z}}$ is a non-linear mapping and $n_{z}$ is the number of outputs.

\section{Probabilistic model of uncertainties for the uncertain positions and orientations}

\subsection{Construction for continuous coordinates}

Let $\underline{\boldsymbol{x}}_{s_{i}}$ in $\Omega$ denote the nominal position of the sensor $s_{i}$ and let $\underline{\boldsymbol{x}}_{a_{i}}$ in $\Omega$ denote the nominal position of the actuator $a_{i}$. Let $\underline{\boldsymbol{x}}$ denote $\underline{\boldsymbol{x}}_{s_{i}}$ or $\underline{\boldsymbol{x}}_{a_{i}}$ and let $\boldsymbol{g}(\underline{\boldsymbol{x}})$ in $\mathbb{C}^{3}$ be a vector depending on the nominal position $\underline{\boldsymbol{x}}$. In the next sections, this vector will represent a displacement measured by a sensor or a force applied by an actuator. It is assumed that the nominal position $\underline{\boldsymbol{x}}$ and the orientation of $\boldsymbol{g}(\underline{\boldsymbol{x}})$ are not perfectly known. Then $\boldsymbol{g}(\underline{\boldsymbol{x}})$ is replaced by the random vector $\boldsymbol{G}(\underline{\boldsymbol{x}})$ with values in $\mathbb{C}^{3}$ such that

$$
\boldsymbol{G}(\underline{\boldsymbol{x}})=[\boldsymbol{R}] \boldsymbol{g}(\underline{\boldsymbol{x}}+\boldsymbol{X}),
$$

in which the random variable $\boldsymbol{X}$, which is such that the random variable $\underline{\boldsymbol{x}}+\boldsymbol{X}$ is with values in $\Omega$, is a random position vector and $[\boldsymbol{R}]$ is a random rotation matrix. There are several possibilities for the construction of the probability models for $\boldsymbol{X}$ and $[\boldsymbol{R}]$.

Concerning the random variation location $\boldsymbol{X}$, the least informative probability distribution is the uniform distribution in a given support $\mathcal{A}$ which is generally centred at the nominal position $\underline{\boldsymbol{x}}$ and is such that for all $\boldsymbol{x}$ in $\mathcal{A}$, $\underline{\boldsymbol{x}}+\boldsymbol{x}$ belongs to $\Omega$. In this case, we have

$$
p_{\boldsymbol{X}}(\boldsymbol{x})=\frac{1}{\operatorname{mes}(\mathcal{A})} \mathbb{1}_{\mathcal{A}}(\boldsymbol{x}),
$$

in which $\operatorname{mes}(\mathcal{A})$ is the measure (volume, surface or length) of support $\mathcal{A}$ and where the indicator function $\boldsymbol{x} \mapsto \mathbb{1}_{\mathcal{A}}(\boldsymbol{x})$ is such that it is equal to 1 
if $\boldsymbol{x} \in \mathcal{A}$ and is zero otherwise. The support $\mathcal{A}$ can be evaluated based on expert judgement and depends on the precision of the placements of the sensors and the actuators. If additional information related to the random position $\boldsymbol{X}$ were available, then more informative probability distributions could be constructed using the Maximum Entropy method [22, 12, 13, 23]. For instance, if information related to the mean value of the components of $\boldsymbol{X}$ were available, then we would obtain a truncated exponential distribution. In addition, if information related to second-order moment of the components of $\boldsymbol{X}$ were also available, then we would obtain a truncated Gaussian distribution.

There are several ways to parametrize rotation matrices: Euler Angles, Euler parameters, Rodriguez parameters, and so on (see for instance [26]). In this paper, we use Euler angles. This parametrization consists in three successive rotations $\theta_{x}, \theta_{y}$ and $\theta_{z}$ around $\boldsymbol{x}$-, $\boldsymbol{y}$ - and $\boldsymbol{z}$-directions (there exist other possible sequences for Euler angles [26]). This parametrization which has a direct interpretation allows an expert judgement to be easily taken into account. Let $\Theta=\left(\Theta_{x}, \Theta_{y}, \Theta_{z}\right)$ be the random rotation vector. The probability distribution of $\Theta$ can be defined as uniform in a given support $\mathcal{B} \subset[-\pi, \pi] \times[-\pi / 2, \pi / 2] \times[-\pi, \pi]$ (for real applications, typical supports are about $\pm 0.2 \mathrm{rad}$ around each direction). In this case, we have

$$
p_{\boldsymbol{\Theta}}(\boldsymbol{x})=\frac{1}{\operatorname{mes}(\mathcal{B})} \mathbb{1}_{\mathcal{B}}(\boldsymbol{\theta}) .
$$

Again, if additional information related to random vector $\Theta$ were available there a more informative probability distribution could be constructed. Then the random matrix $[\boldsymbol{R}]$ is such that

$$
[\boldsymbol{R}]=\left[\begin{array}{ccc}
C_{y} C_{z} & -S_{z} C_{x} & S_{y} \\
S_{z} C_{x}+S_{x} S_{y} C_{z} & C_{z} C_{x}-S_{x} S_{y} S_{z} & -S_{x} C_{y} \\
S_{x} S_{z}-C_{x} S_{y} C_{z} & S_{x} C_{z}+C_{x} S_{y} S_{z} & C_{x} C_{y}
\end{array}\right]
$$

in which $C_{x}=\cos \left(\Theta_{x}\right), C_{y}=\cos \left(\Theta_{y}\right), C_{z}=\cos \left(\Theta_{z}\right), S_{x}=\sin \left(\Theta_{x}\right), S_{y}=$ $\sin \left(\Theta_{y}\right)$ and $S_{z}=\sin \left(\Theta_{z}\right)$.

\subsection{Discretization of the probabilistic model of uncertainties}

In the context of the FE method, the displacement $\boldsymbol{u}(\boldsymbol{x}, \omega)$ is approximated for $\boldsymbol{x}$ in $\Omega$ by

$$
\boldsymbol{u}(\boldsymbol{x}, \omega)=[N(\boldsymbol{x})] \mathbb{U}(\omega)
$$


in which $[N(\underline{\boldsymbol{x}})]$ is the matrix of the shape functions. The displacement vector $\mathbb{U}^{\text {sens,i }}(\omega)=\boldsymbol{u}\left(\underline{\boldsymbol{x}}_{s_{i}}, \omega\right)$ of the sensor $s_{i}$ is replaced by the random vector $\boldsymbol{U}^{\text {sens,i }}(\omega)$. Then using Eq.(10), we have

$$
\boldsymbol{U}^{\mathrm{sens, \textrm {i }}}(\omega)=\left[\boldsymbol{R}_{s_{i}}\right]\left[N\left(\underline{\boldsymbol{x}_{s_{i}}}+\boldsymbol{X}_{s_{i}}\right)\right] \mathbb{U}(\omega) .
$$

Then, by assuming that the FE mesh is fine enough, the random displacement $\boldsymbol{U}^{\text {sens,i }}(\omega)$ can be approximated by

$$
\boldsymbol{U}^{\text {sens,i }}(\omega) \simeq\left[\boldsymbol{R}_{s_{i}}\right]\left[\overline{\boldsymbol{O}}_{s_{i}}\right] \mathbb{U}(\omega),
$$

in which $\left[\overline{\boldsymbol{O}}_{s_{i}}\right]$ is a $(3 \times m)$ random localization matrix that associates the DOFS of the closest FE node from position $\boldsymbol{x}_{s_{i}}+\boldsymbol{X}_{s_{i}}$ to the DOFs of the FE mesh. With such an approximation, we only need the coordinates of the nodes of the mesh yielding little intrusiveness with respect to commercial softwares. Finally, the random displacement $\boldsymbol{U}^{\text {sens,i }}(\omega)$ can be written as

$$
\boldsymbol{U}^{\mathrm{sens, \textrm {i }}}(\omega)=\left[\boldsymbol{O}_{s_{i}}\right] \mathbb{U}(\omega),
$$

in which $\left[\boldsymbol{O}_{s_{i}}\right]=\left[\boldsymbol{R}_{s_{i}}\right]\left[\overline{\boldsymbol{O}}_{s_{i}}\right]$ is a $(3 \times m)$ random matrix.

Concerning the actuators, let $\Gamma$ be the part of the boundary of the structure where forces (possibly zero) are applied. Then for any position $\boldsymbol{x}$ on $\Gamma$, the force field $\boldsymbol{f}\left(\boldsymbol{x}, \omega ; \underline{\boldsymbol{x}_{a_{i}}}\right)$ induced by the point force $\mathbb{F}^{\text {act,i }}(\omega)$ applied at $\underline{\boldsymbol{x}_{a_{i}}}$ is such that

$$
\boldsymbol{f}\left(\boldsymbol{x}, \omega ; \underline{\boldsymbol{x}}_{a_{i}}\right)=\delta\left(\boldsymbol{x}-\underline{\boldsymbol{x}}_{a_{i}}\right) \mathbb{F}^{\text {act,i }}(\omega),
$$

where $\boldsymbol{x} \mapsto \delta(\boldsymbol{x})$ is the surface Dirac distribution such that for any continuous function $\boldsymbol{h}(\boldsymbol{x})$ we have $\int_{\Gamma} \delta\left(\boldsymbol{x}-\boldsymbol{x}_{a_{i}}\right) \boldsymbol{h}(\boldsymbol{x}) d s(\boldsymbol{x})=\boldsymbol{h}\left(\boldsymbol{x}_{a_{i}}\right)$. Using Eq. (10), the corresponding random force field $\overline{\boldsymbol{F}}\left(\boldsymbol{x}, \omega ; \underline{\boldsymbol{x}}_{a_{i}}\right)$ is written as

$$
\boldsymbol{F}\left(\boldsymbol{x}, \omega ; \underline{\boldsymbol{x}}_{a_{i}}\right)=\delta\left(\boldsymbol{x}-\left(\underline{\boldsymbol{x}_{a_{i}}}+\boldsymbol{X}_{a_{i}}\right)\right)\left[\boldsymbol{R}_{a_{i}}\right] \mathbb{F}^{\mathrm{act}, \mathrm{i}}(\omega) .
$$

The corresponding random force vector $\boldsymbol{F}^{i}(\omega)$ related to the FE discretization is such that

$$
\boldsymbol{F}^{i}(\omega)=\int_{\Gamma}[N(\boldsymbol{x})]^{T} \boldsymbol{F}\left(\underline{\boldsymbol{x}_{a_{i}}}, \omega ; \boldsymbol{x}\right) d s(\boldsymbol{x}),
$$

which can be rewritten as

$$
\boldsymbol{F}^{i}(\omega)=\left[N\left(\underline{\boldsymbol{x}_{a_{i}}}+\boldsymbol{X}_{a_{i}}\right)\right]^{T}\left[\boldsymbol{R}_{a_{i}}\right] \mathbb{F}^{\mathrm{act}, \mathrm{i}}(\omega)
$$


and which can finally be approximated by

$$
\boldsymbol{F}^{i}(\omega) \simeq\left[\overline{\boldsymbol{C}}_{a_{i}}\right]\left[\boldsymbol{R}_{a_{i}}\right] \mathbb{F}^{\text {act,i }}(\omega),
$$

in which $\left[\overline{\boldsymbol{C}}_{a_{i}}\right]$ is a $(m \times 3)$ random localization matrix that associates the DOFS of the closest FE node from position $\boldsymbol{x}_{a_{i}}+\boldsymbol{X}_{a_{i}}$ to the DOFs of the FE mesh. Finally, the random force vector $\overline{\boldsymbol{F}^{i}(\omega)}$ can be written as

$$
\boldsymbol{F}^{i}(\omega)=\left[\boldsymbol{C}_{a_{i}}\right] \mathbb{F}^{\text {act,i }}(\omega),
$$

in which $\left[\boldsymbol{C}_{a_{i}}\right]=\left[\overline{\boldsymbol{C}}_{a_{i}}\right]\left[\boldsymbol{R}_{a_{i}}\right]$ is a $(m \times 3)$ random matrix.

Finally for all $\omega$ in $B$, the observed random displacement vector $\boldsymbol{U}^{\text {sens }}(\omega)$ is written as

$$
\boldsymbol{U}^{\text {sens }}(\omega)=[\boldsymbol{O}][H(\omega)][\boldsymbol{C}] \mathbb{F}^{\text {act }}(\omega),
$$

in which the $\left(n_{\text {obs }} \times m\right)$ random localization matrix $[\boldsymbol{O}]$ and the $\left(m \times n_{\text {exc }}\right)$ random localization matrix $[\boldsymbol{C}]$ are obtained by assembling the random matrices $\left[\boldsymbol{O}_{s_{1}}\right], \ldots,\left[\boldsymbol{O}_{s_{n_{s}}}\right]$ and the random matrices $\left[\boldsymbol{C}_{a_{1}}\right], \ldots,\left[\boldsymbol{C}_{a_{n_{a}}}\right]$ respectively. It is interesting to compare Eq. (24) to Eq. (7). Finally, the deterministic localization matrices $[O]$ and $[C]$ are just replaced by random localization matrices $[\boldsymbol{O}]$ and $[\boldsymbol{C}]$. Therefore the calculation of independent realizations of the random displacement $\boldsymbol{U}^{\text {sens }}(\omega)$ is little intrusive with respect to commercial softwares. Furthermore, once the elastic modes have been calculated initially using the nominal computational model, no additional eigenvalue problem has to be solved to calculate the random response of the model. Only calculations at the reduced-order level have to be performed.

The output vector $\boldsymbol{z}$ becomes a random vector $\boldsymbol{Z}=\hat{\boldsymbol{z}}\left(\boldsymbol{U}^{\text {sens }}\left(\omega_{1}\right), \ldots\right.$, $\left.\boldsymbol{U}^{\text {sens }}\left(\omega_{n_{\text {freq }}}\right)\right)$ with values in $\mathbb{R}^{n_{z}}$.

\section{Updating of the parameters}

Let $\boldsymbol{h}$ be the vector of the $n_{h}$ parameters to be updated. This parameters can be related to mass, stiffness, damping or geometry properties. Let $\mathcal{C}_{h}$ be the admissible set for the vector $\boldsymbol{h}$. Then for all $\boldsymbol{h}$ in $\mathcal{C}_{h}$, Eq. (24) is rewritten as

$$
\boldsymbol{U}^{\text {sens }}(\omega, \boldsymbol{h})=[\boldsymbol{O}][H(\omega, \boldsymbol{h})][\boldsymbol{C}] \mathbb{F}^{\text {act }}(\omega),
$$

and then

$$
\boldsymbol{Z}(\boldsymbol{h})=\boldsymbol{f}_{z}\left(\boldsymbol{U}^{\mathrm{sens}}\left(\omega_{1}, \boldsymbol{h}\right), \ldots, \boldsymbol{U}^{\mathrm{sens}}\left(\omega_{n_{\mathrm{freq}}}, \boldsymbol{h}\right)\right)
$$


The uncertainties induced by modeling errors and measurement errors (except sensor and actuator placement errors) are modeled by an output prediction error $\boldsymbol{N}$ which is assumed to be a centred Gaussian random vector with covariance matrix $\left[C_{N}\right]$, which has also to be updated or is directly given based on expert judgement. For readability purpose, it is assumed that the covariance matrix $\left[C_{N}\right]$ of the output error is given and therefore is not to be updated. The generalization of the hereinafter derived equations to the case of a covariance matrix to be updated is straightforward $[2,3,17]$. Then the observed random $\boldsymbol{Z}^{\text {obs }}$ is defined by

$$
Z^{\text {obs }}=Z(\boldsymbol{h})+N
$$

Let $\boldsymbol{z}^{\exp }$ be the measured vector corresponding to random output vector $\boldsymbol{Z}^{\text {obs }}$. The Bayesian updating method $[9,2,3]$ consists in replacing the deterministic parameter $\boldsymbol{h}$ by the random variable $\boldsymbol{H}^{\text {prior }}$ with values in $\mathcal{C}_{h}$ and for which the prior probability distribution $p_{\boldsymbol{H}^{\text {prior }}}(\boldsymbol{h})$ is given. For any random variable $\boldsymbol{X}$, let $E_{\boldsymbol{X}}$ denote the mathematical expectation with respect to $\boldsymbol{X}$. Then the posterior distribution $p_{\boldsymbol{H}^{\text {post }}}\left(\boldsymbol{h} \mid \boldsymbol{z}^{\exp }\right)$ is given by

$$
p_{\boldsymbol{H}^{\text {post }}}\left(\boldsymbol{h} \mid \boldsymbol{z}^{\exp }\right)=\frac{1}{c} p_{\boldsymbol{Z}^{\mathrm{obs}} \mid \boldsymbol{H}^{\text {prior }}}\left(\boldsymbol{z}^{\exp } \mid \boldsymbol{h}\right) \times p_{\boldsymbol{H}^{\text {prior }}}(\boldsymbol{h}),
$$

in which $c=E_{\boldsymbol{H}^{\text {prior }}}\left\{p_{\boldsymbol{Z}^{\text {obs }} \mid \boldsymbol{H}^{\text {prior }}}\left(\boldsymbol{z}^{\exp } \mid \boldsymbol{H}^{\text {prior }}\right)\right\}$ is a normalization constant. For computational purpose, the likelihood function $p_{\boldsymbol{Z}^{\text {obs }}} \boldsymbol{H}^{\text {prior }}\left(\boldsymbol{z}^{\exp } \mid \boldsymbol{h}\right)$ is rewritten as follow

$$
p_{\boldsymbol{Z}^{\text {obs }} \mid \boldsymbol{H}^{\text {prior }}}\left(\boldsymbol{z}^{\exp } \mid \boldsymbol{h}\right)=E_{\boldsymbol{N}}\left\{p_{\boldsymbol{Z}^{\text {obs }} \mid \boldsymbol{N}, \boldsymbol{H}^{\text {prior }}}\left(\boldsymbol{z}^{\exp } \mid \boldsymbol{N}, \boldsymbol{h}\right)\right\} .
$$

From Eq.(27), it can be deduced that

$$
p_{\boldsymbol{Z}^{\text {obs }} \mid \boldsymbol{N}, \boldsymbol{H}^{\text {prior }}}\left(\boldsymbol{z}^{\exp } \mid \boldsymbol{n}, \boldsymbol{h}\right)=p_{\boldsymbol{Z} \mid \boldsymbol{H}^{\text {prior }}}\left(\boldsymbol{z}^{\exp }-\boldsymbol{n} \mid \boldsymbol{h}\right) .
$$

Then the posterior distribution $p_{\boldsymbol{H}^{\text {post }}}\left(\boldsymbol{h} \mid \boldsymbol{z}^{\exp }\right)$ can be rewritten as

$$
p_{\boldsymbol{H}^{\text {post }}}\left(\boldsymbol{h} \mid \boldsymbol{z}^{\exp }\right)=\frac{\ell\left(\boldsymbol{h}, \boldsymbol{z}^{\exp }\right)}{E_{\boldsymbol{H}^{\text {prior }}}\left\{\ell\left(\boldsymbol{H}^{\text {prior }}, \boldsymbol{z}^{\exp }\right)\right\}} \times p_{\boldsymbol{H}^{\text {prior }}}(\boldsymbol{h}),
$$

where $\ell\left(\boldsymbol{h}, \boldsymbol{z}^{\exp }\right)=E_{\boldsymbol{N}}\left\{p_{\boldsymbol{Z} \mid \boldsymbol{H}^{\text {prior }}}\left(\boldsymbol{z}^{\exp }-\boldsymbol{N} \mid \boldsymbol{h}\right)\right\}$ is the likelihood function. Then several estimators $\hat{\boldsymbol{h}}$ of the parameter $\boldsymbol{h}$ can be constructed. This esti- 
mation can be chosen as the value that maximizes the posterior distribution $p_{\boldsymbol{H}^{\text {post }}}\left(\boldsymbol{h} \mid \boldsymbol{z}^{\exp }\right)$ yielding the maximum a posteriori probability estimation method:

$$
\hat{\boldsymbol{h}}=\arg \max _{\boldsymbol{h} \in \mathcal{C}_{h}} \ell\left(\boldsymbol{h}, \boldsymbol{z}^{\exp }\right) p_{\boldsymbol{H}^{\text {prior }}}(\boldsymbol{h}) .
$$

The optimization problem defined by Eq. (32) is, in general, non-convex. If the number $n_{h}$ of updating parameters is large, the maximum can be search using a random search algorithm such as an evolutionary algorithm $[25,8,24]$. If the prior probability distribution $p_{\boldsymbol{H}^{\text {prior }}}(\boldsymbol{h})$ is uniform on the support $\mathcal{C}_{h}$ then the estimator (32) becomes a maximum likelihood estimator. The estimate $\hat{\boldsymbol{h}}$ can also be constructed as the mean values of random variable $\boldsymbol{H}^{\text {post }}$, i.e

$$
\hat{\boldsymbol{h}}=E_{\boldsymbol{H}^{\text {post }}}\left\{\boldsymbol{H}^{\text {post }}\right\}=\frac{E_{\boldsymbol{H}^{\text {prior }}}\left\{\boldsymbol{H}^{\text {prior }} \ell\left(\boldsymbol{H}^{\text {prior }}, \boldsymbol{z}^{\exp }\right)\right\}}{E_{\boldsymbol{H}^{\text {prior }}}\left\{\ell\left(\boldsymbol{H}^{\text {prior }}, \boldsymbol{z}^{\exp }\right)\right\}},
$$

In the context of Monte Carlo estimation [20], this latter rewriting only requires independent realizations of random variable $\boldsymbol{H}^{\text {prior }}$, which are easier to obtain than independent realizations of random variable $\boldsymbol{H}^{\text {post }}$ for which the generation would require the use of a Monte Carlo Markov Chain (MCMC) method. Let $\boldsymbol{h}^{\text {prior }, 1}, \ldots, \boldsymbol{h}^{\text {prior, } n_{\text {sim }, h}}$ be $n_{\text {sim }, h}$ independent realizations of random variable $\boldsymbol{h}^{\text {prior }}$ and let $\boldsymbol{n}^{1}, \ldots, \boldsymbol{n}^{n_{\text {sim }, n}}$ be $n_{\text {sim }, n}$ independent realizations of random variable $\boldsymbol{N}$. Then the estimation $\hat{\boldsymbol{h}}_{n_{s i m, h}}$ related to Eq. (33) is constructed using the Monte Carlo simulation method, i.e.,

$$
\hat{\boldsymbol{h}}_{n_{\text {sim }, h}}=\frac{\sum_{i=1}^{n_{\text {sim }, h}} \boldsymbol{h}^{\text {prior }, i} \hat{\ell}\left(\boldsymbol{h}^{\text {prior }, i}, \boldsymbol{z}^{\exp }\right)}{\sum_{i=1}^{n_{\text {sim, }, h}} \hat{\ell}\left(\boldsymbol{h}^{\text {prior }, i}, \boldsymbol{z}^{\exp }\right)}
$$

where the function $\hat{\ell}\left(\boldsymbol{h}, \boldsymbol{z}^{\exp }\right)$ is an estimation of the likelihood function such that

$$
\hat{\ell}\left(\boldsymbol{h}, \boldsymbol{z}^{\exp }\right)=\frac{1}{n_{\text {sim }, n}} \sum_{k=1}^{n_{\text {sim }, n}} \hat{p}_{\boldsymbol{Z} \mid \boldsymbol{H}^{\text {prior }}}\left(\boldsymbol{z}^{\exp }-\boldsymbol{n}^{k} \mid \boldsymbol{h}\right),
$$

where $\hat{p}_{\boldsymbol{Z} \mid \boldsymbol{H}^{\text {prior }}}$ is a multivariate kernel density estimator [27] of the probability distribution $p_{\boldsymbol{Z} \mid \boldsymbol{H}^{\text {prior }}}$ which is constructed using $n_{\text {sim,p }, p}$ independent realizations of the random localization matrices $[\boldsymbol{O}]$ and $[\boldsymbol{C}]\left(\boldsymbol{H}^{\text {prior }}\right.$ being fixed). It is recalled that, for each realization of $\boldsymbol{H}^{\text {prior }}$, the calculation of the elastic modes, which is time-consuming has to be calculated only one time. Then the 
calculation of the independent realizations of random variable $\boldsymbol{Z}$ given $\boldsymbol{H}^{\text {prior }}$, which are useful for estimating $p_{\boldsymbol{Z} \mid \boldsymbol{H}^{\text {prior }}}$ using a kernel density estimator are not time-consuming. The computational cost related to the expectation with respect to random variable $N$ is also negligible once the probability distribution $p_{\boldsymbol{Z} \mid \boldsymbol{H}^{\text {prior }}}$ is estimated. In case of large dimension for random variable $\boldsymbol{Z}$, the estimation of $p_{\boldsymbol{Z} \mid \boldsymbol{H}^{\text {prior }}}$ using a multivariate kernel density estimator becomes inaccurate. To improve the accuracy, we can introduce the random variable $\widetilde{\boldsymbol{Z}}=[V]^{-1}(\boldsymbol{Z}-E\{\boldsymbol{Z}\})$, in which $[V]$ is related to the Cholesky factorization of the covariance matrix $\left[C_{\boldsymbol{Z}}\right]=E\left\{(\boldsymbol{Z}-E\{\boldsymbol{Z}\})\left(\boldsymbol{Z}-E\{\boldsymbol{Z})^{T}\right\}\right.$, i.e, $\left[C_{\boldsymbol{Z}}\right]=[V][V]^{T}$. Then it can be verified that the components of random vector $\widetilde{Z}$ are uncorrelated but dependent and we have the relation

$$
p_{\boldsymbol{Z} \mid \boldsymbol{H}^{\text {prior }}}(\boldsymbol{z} \mid \boldsymbol{h})=\frac{1}{\operatorname{det}([V])} p_{\widetilde{\boldsymbol{Z}} \mid \boldsymbol{H}^{\text {prior }}}\left([V]^{-1}(\boldsymbol{z}-E\{\boldsymbol{Z}\}) \mid \boldsymbol{h}\right) .
$$

Since the construction the components of random vector $\widetilde{\boldsymbol{Z}}$ are uncorrelated, a multivariate kernel density estimator $\hat{p}_{\widetilde{\boldsymbol{Z}} \mid \boldsymbol{H}^{\text {prior }}}$ for $\widetilde{\boldsymbol{Z}} \mid \boldsymbol{H}^{\text {prior }}$ can be constructed with a good accuracy. Then a multivariate kernel density estimator $\hat{p}_{\boldsymbol{Z} \mid \boldsymbol{H}^{\text {prior }}}$ for random variable $\boldsymbol{Z} \mid \boldsymbol{H}^{\text {prior }}$ can be constructed using Eq. (36).

\section{Optimal sensor location}

The objective now is to determine the optimal nominal locations $\underline{\boldsymbol{x}}_{s_{1}}, \ldots$, $\underline{\boldsymbol{x}}_{s_{n_{s}}}$ and $\underline{\boldsymbol{x}}_{a_{1}}, \ldots, \underline{\boldsymbol{x}}_{a_{a_{s}}}$ for the sensors and actuators respectively. A review of previous methodologies for optimal sensors/actuators placements in linear and non-linear structural dynamics can be found for instance in [18, 5, 19]. In the present paper, the optimal positions are searched in order to collect the maximum information for updating the parameter $\boldsymbol{h}$. This means that we want that the dynamical responses measured at the optimal positions should be (1) as sensitive as possible with respect to the parameter $\boldsymbol{h}$ and (2) as robust as possible with respect to positioning uncertainties (and to modeling errors if they are not negligible). In $[16,17,18]$, the authors have proposed to use the Shannon differential entropy $[22,12,13]$ to quantify the information that is provided by each sensor/actuator configuration. More precisely, this method consists in minimizing the entropy (or uncertainty) related to the posterior probability distribution $p_{\boldsymbol{H}^{\text {post }}}$. In the present paper, the Kullback-Leibler (KL) divergence [14, 13], which is commonly used in Bayesian experimental design (and more specifically for optimal sensor 
placement $[6,28])$ and which is the opposite of the Shannon entropy in case of uniform prior, is used instead. Let $\underline{\boldsymbol{x}}_{s, a}=\left(\underline{\boldsymbol{x}}_{s_{1}}, \ldots, \underline{\boldsymbol{x}}_{s_{n_{s}}}, \underline{\boldsymbol{x}}_{a_{1}}, \ldots, \underline{\boldsymbol{x}}_{a_{a_{s}}}\right)$ and let $\mathcal{C}_{\underline{\boldsymbol{x}}_{s, a}}$ be the admissible space for $\underline{\boldsymbol{x}}_{s, a}$. The KL divergence between the prior distribution $p_{\boldsymbol{H}^{\text {prior }}}$ and the posterior distribution $p_{\boldsymbol{H}^{\text {post }}}$ is defined by

$$
\begin{aligned}
\mathcal{K} \mathcal{L}\left(p_{\boldsymbol{H}^{\text {post }}}, p_{\boldsymbol{H}^{\text {prior }}}\right)=\int_{\mathbb{R}^{n_{h}}} p_{\boldsymbol{H}^{\text {post }}}(\boldsymbol{h}) & \log \left(\frac{p_{\boldsymbol{H}^{\text {post }}}(\boldsymbol{h})}{p_{\boldsymbol{H}^{\text {prior }}}(\boldsymbol{h})}\right) d \boldsymbol{h} \\
& =E_{\boldsymbol{H}^{\text {post }}}\left\{\log \left(\frac{p_{\boldsymbol{H}^{\text {post }}}\left(\boldsymbol{H}^{\text {post }}\right)}{p_{\boldsymbol{H}^{\text {prior }}}\left(\boldsymbol{H}^{\text {post }}\right)}\right)\right\},
\end{aligned}
$$

where $\log$ is the natural logarithm. The KL divergence is non-negative and measures the difference of quantity of information between $p_{\boldsymbol{H}^{\text {prior }}}$ and $p_{\boldsymbol{H}^{\text {post }}}$. This KL divergence depends on the measured vector $\boldsymbol{z}^{\exp }$ which is not available during the design of the experimental configuration. Therefore the experimental vector has to be constructed numerically using for instance the response $\boldsymbol{z}^{\text {nom }}$ of the nominal model for which the updating parameter $\boldsymbol{h}$ is set to a nominal value $\boldsymbol{h}^{\text {nom }}$. Using Eq. (31) with $\boldsymbol{z}^{\exp }=\boldsymbol{z}^{\text {nom }}$, the KL divergence is rewritten as

$$
\begin{aligned}
& \mathcal{K} \mathcal{L}\left(p_{\boldsymbol{H}^{\text {post }}}, p_{\boldsymbol{H}^{\text {prior }}}\right)=E_{\boldsymbol{H}^{\text {prior }}}\left\{\log \left(\frac{\ell\left(\boldsymbol{H}^{\text {prior }}, \boldsymbol{z}^{\text {nom }}\right)}{E_{\boldsymbol{H}^{\text {prior }}}\left\{\ell\left(\boldsymbol{H}^{\text {prior }}, \boldsymbol{z}^{\text {nom }}\right)\right\}}\right)\right. \\
& \left.\times \frac{\ell\left(\boldsymbol{H}^{\text {prior }}, \boldsymbol{z}^{\text {nom }}\right)}{E_{\boldsymbol{H}^{\text {prior }}}\left\{\ell\left(\boldsymbol{H}^{\text {prior }}, \boldsymbol{z}^{\text {nom }}\right)\right\}}\right\} \\
& =\frac{E_{\boldsymbol{H}^{\text {prior }}}\left\{\log \left(\ell\left(\boldsymbol{H}^{\text {prior }}, \boldsymbol{z}^{\text {nom }}\right)\right) \ell\left(\boldsymbol{H}^{\text {prior }}, \boldsymbol{z}^{\text {nom }}\right)\right\}}{E_{\boldsymbol{H}^{\text {prior }}}\left\{\ell\left(\boldsymbol{H}^{\text {prior }}, \boldsymbol{z}^{\text {nom }}\right)\right\}} \\
& -\log \left(E_{\boldsymbol{H}^{\text {prior }}}\left\{\ell\left(\boldsymbol{H}^{\text {prior }}, \boldsymbol{z}^{\text {nom }}\right)\right\}\right) \text {. }
\end{aligned}
$$

Here again, this rewriting has the advantage to use a generator of realizations of $\boldsymbol{H}^{\text {prior }}$ instead of $\boldsymbol{H}^{\text {post }}$. Then the KL divergence can be estimated using the Monte Carlo simulation method, i.e.,

$$
\begin{array}{r}
\widehat{\mathcal{K L}}\left(p_{\boldsymbol{H}^{\text {post }}}, p_{\boldsymbol{H}^{\text {prior }}}\right)=\frac{\sum_{i=1}^{n_{\text {sim }, h}} \log \left(\hat{\ell}\left(\boldsymbol{h}^{\text {prior }, i}, \boldsymbol{z}^{\mathrm{nom}}\right)\right) \hat{\ell}\left(\boldsymbol{h}^{\text {prior }, i}, \boldsymbol{z}^{\mathrm{nom}}\right)}{\sum_{i=1}^{n_{\text {sim }, h}} \hat{\ell}\left(\boldsymbol{h}^{\text {prior }, i}, \boldsymbol{z}^{\text {nom }}\right)} \\
-\log \left(\frac{1}{n_{\text {sim }, h}} \sum_{i=1}^{n_{\text {sim }, h}} \hat{\ell}\left(\boldsymbol{h}^{\text {prior }, i}, \boldsymbol{z}^{\text {nom }}\right)\right),
\end{array}
$$


in which the estimated likelihood function $\hat{\ell}\left(\boldsymbol{h}, \boldsymbol{z}^{\text {nom }}\right)$ is given by Eq. (35). The computation of the KL divergence for a given sensor/actuator configuration $\underline{\boldsymbol{x}}_{s, a}$ is summarized on Table 1 .

Choose a sensor/actuator configuration $\underline{\boldsymbol{x}}_{s, a} \in \mathcal{C}_{\underline{\boldsymbol{x}}_{s, a}}$.

Calculate $\boldsymbol{z}^{\text {nom }}$ using Eqs. (7) and (9).

for $i=1, \ldots, n_{\text {sim }, h}$ do

Generate realization $\boldsymbol{h}^{\text {prior,i }}$ of random vector $\boldsymbol{H}^{\text {prior }}$.

for $j=1, \ldots, n_{\text {sim }, p}$ do

Generate realization $\left[O^{j}\right]$ of random matrix $[\boldsymbol{O}]$.

Generate realization $\left[C^{j}\right]$ of random matrix $[\boldsymbol{C}]$.

Calculate $\boldsymbol{z}\left(\boldsymbol{h}^{\text {prior, } i} ;\left[O^{j}\right],\left[C^{j}\right]\right)$ using Eqs. (25) and (26).

Estimate the probability distribution $\boldsymbol{z} \mapsto \hat{p}_{\boldsymbol{Z} \mid \boldsymbol{H}^{\text {prior }}}\left(\boldsymbol{z} \mid \boldsymbol{h}^{\text {prior,i }}\right)$ using a kernel density estimator.

for $k=1, \ldots, n_{\text {sim }, n}$ do

Generate realization $\boldsymbol{n}^{k}$ of the noise vector $\boldsymbol{N}$.

Evaluate $p_{\boldsymbol{Z} \mid \boldsymbol{H}^{\text {prior }}}\left(\boldsymbol{z}^{\exp }-\boldsymbol{n}^{j} \mid \boldsymbol{h}^{\text {prior }, i}\right)$.

Estimate $\hat{\ell}\left(\boldsymbol{h}, \boldsymbol{z}^{\exp }\right)$ using Eq. (35).

Estimate $\widehat{\mathcal{K} \mathcal{L}}\left(p_{\boldsymbol{H}^{\text {post }}}, p_{\boldsymbol{H}^{\text {prior }}} ; \underline{\boldsymbol{x}}_{s, a}\right)$ using Eq. (39).

Table 1: Computation of the KL divergence.

The optimal value $\underline{\boldsymbol{x}}_{s, a}^{\text {opt }}$ for the sensor/actuator nominal locations is the one that maximizes the KL divergence (quantity of information) and is therefore solution of the following optimization problem

$$
\underline{\boldsymbol{x}}_{s, a}^{\text {opt }}=\underset{\underline{\boldsymbol{x}}_{s, a} \in \underline{\mathcal{C}}_{s, a}}{\arg \max } \mathcal{K} \mathcal{L}\left(p_{\boldsymbol{H}^{\text {post }}}, p_{\boldsymbol{H}^{\text {prior }}} ; \underline{\boldsymbol{x}}_{s, a}\right) .
$$

This optimization problem can be solved using an evolutionary algorithm. In [17], a sequential algorithm has been proposed in order to place the sensors one at a time.

\section{Remarks:}

- The most extensive steps are related to the computation of the modal bases $\left[\Phi\left(\boldsymbol{h}^{\text {prior }, 1}\right)\right], \ldots,\left[\Phi\left(\boldsymbol{h}^{\text {prior }, n_{\text {sim }, h}}\right)\right]$ for each sensor/actuator configuration. In 
order to save computational time, the same realizations $h^{\text {prior }, 1}, \ldots, h^{\text {prior }, n_{\text {sim }, h}}$ of the random vector $H^{\text {prior }}$ and the same realizations of the modal bases $\left[\Phi\left(\boldsymbol{h}^{\text {prior }, 1}\right)\right], \ldots,\left[\Phi\left(\boldsymbol{h}^{\text {prior }, n_{\text {sim }, h}}\right)\right]$ can be used for each configuration. Nevertheless, this pre-computation of the modal bases requires a lot of memory space available.

- The convergence of the KL divergence $\widehat{\mathcal{K L}}\left(p_{\boldsymbol{H}^{\text {post }}}, p_{\boldsymbol{H}^{\text {prior }}} ; \underline{\boldsymbol{x}}_{s, a}\right)$ with respect to $n_{s i m, h}, n_{s i m, p}$ and $n_{s i m, n}$ should be studied for each sensor/actuator configuration or at least for the nominal sensor/actuator configuration.

\section{Application}

\subsection{D-Application: uniform bar}

This first application is related to the frequency response of a uniform bar with length $L=1 \mathrm{~m}$, section $S=10^{-3} \mathrm{~m}^{2}$, Young's modulus $E=10^{9} \mathrm{~Pa}$, mass density $\rho=9000 \mathrm{~kg} / \mathrm{m}^{3}$.

\subsubsection{Reduced-order model and experimental responses}

For such a simple structure, the elastic modes can be calculated explicitly. For $\alpha \in \mathbb{N}^{*}$, the eigenfrequencies $\lambda_{\alpha}=\alpha^{2} \pi^{2} E /\left(\rho L^{2}\right)$ are associated with the mass-normalized elastic modes $\phi_{\alpha}=2 \sin (\alpha \pi x / L) / L, x \in[0, L]$. A unit point force is applied at $x=\underline{x}_{a}$ such that the corresponding generalized forces are $f_{\alpha}=2 \sin \left(\alpha \pi x_{a} / L\right) / L$. In a first stage, in order to construct the experimental responses, the modal dampings $\xi_{\alpha}$ are equal to 0.04. The frequency band of analysis is $B_{1}=[0 ; 3250] \times 2 \pi \mathrm{rad} / \mathrm{s}$. The reduced-order model is constructed using the 15 first elastic modes in order to a have a good convergence of the response in the band $B_{1}$. The frequency response is calculated at $\underline{x}_{a}=0.3 \mathrm{~m}$ and for observation points $P 1$ and $P 2$ located at $\underline{x}_{s}=0.5 \mathrm{~m}$ and $\underline{x}_{s}=0.25 \mathrm{~m}$ respectively. The functions $\omega \mapsto \log _{10}\left(\omega\left|\mathbb{U}^{\text {sens, }}, \mathrm{P}_{1}(\omega)\right|\right)$ and $\omega \mapsto \log _{10}\left(\omega\left|\mathbb{U}^{\text {sens, }} \mathrm{P}_{2}(\omega)\right|\right)$ are plotted on Figure 1. As expected, the resonance corresponding to the second elastic mode $(166 \mathrm{~Hz})$ vanishes for point $P 1$ and the resonance corresponding to the fourth elastic mode $(667 \mathrm{~Hz})$ vanishes for points $P 1$ and $P 2$.

\subsubsection{Probabilistic model of positioning uncertainties and random response}

Since the problem is 1D (no rotation), we only have to consider uncertainties for the positions $\underline{x}_{s}$ and $\underline{x}_{a}$ related to the observation point and the excitation point respectively. These positions are replaced by the random positions $\underline{x}_{s}+X_{s}$ and $\underline{x}_{a}+X_{a}$ in which random variables $X_{s}$ and $X_{a}$ are uniform 

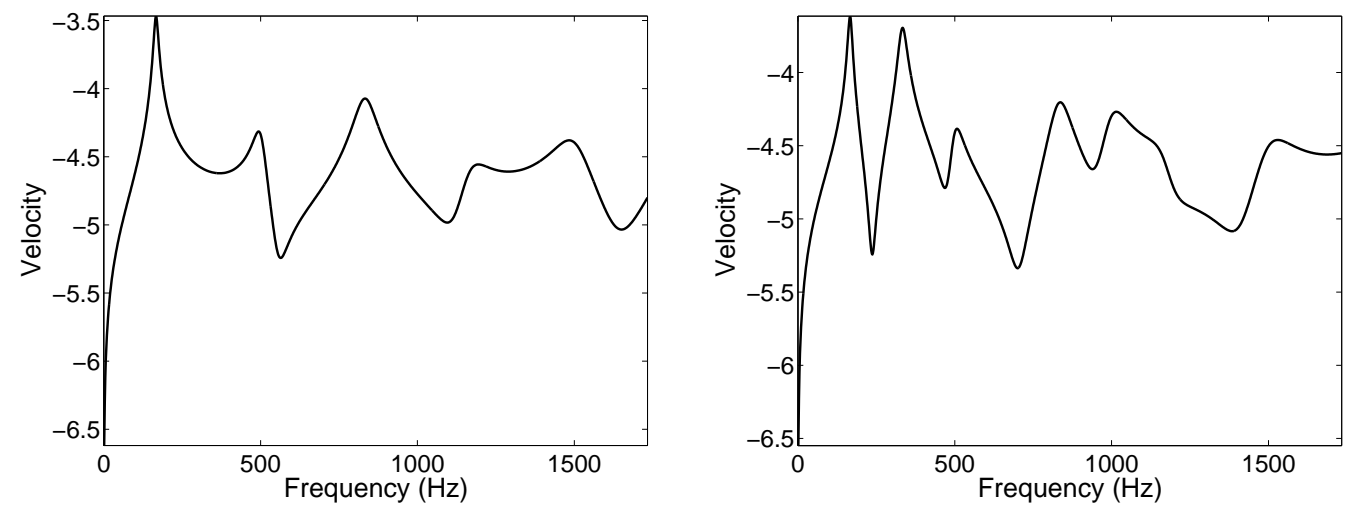

Figure 1: Frequency response in velocity for points $P 1$ (left figure) and $P 2$ (right figure).

random variables defined on support $[-0.04,0.04]$. For $\underline{x}_{s}=0.5 \mathrm{~m}$ (point $P_{1}$ ) and $\underline{x}_{s}=0.25 \mathrm{~m}$ (point $P_{2}$ ), for $\underline{x}_{a}=0.3 \mathrm{~m}$, the random frequency responses are plotted on Fig. 2. The confidence regions with probability level 0.9 and the mean values are estimated using the Monte Carlo simulation method with 1000 realizations. The convergence of the L2-norm of the mean value with respect to the number of Monte Carlo simulations is plotted on Fig. 3. It
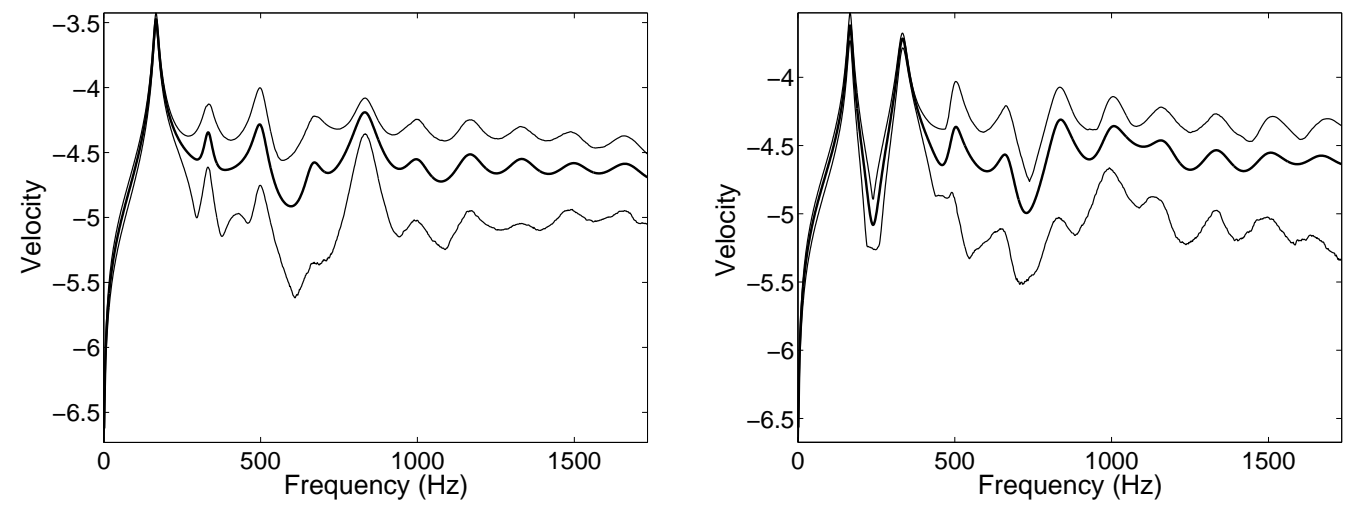

Figure 2: Random frequency response in velocity for points $P 1$ (left figure) and $P 2$ (right figure): Mean response (thick line) and confidence region (thin lines).

can be seen on Fig. 2 that compared to Fig. 1, a random resonance related to the second elastic mode appears for point $P 1$ and a random resonance related to the fourth elastic mode appears for points $P 1$ and $P 2$. It can also be seen 


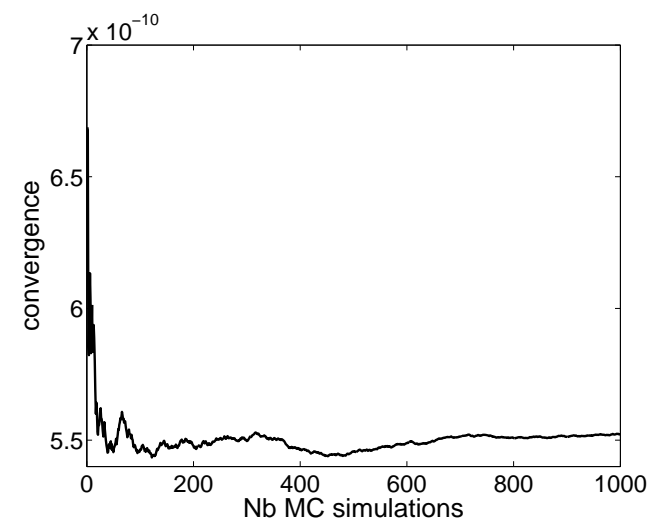

Figure 3: Convergence of the mean value with respect to the number of Monte Carlo simulations.

that the level of fluctuation related to the first resonance mode is higher for point $P 2$ and the level of fluctuation related to the second resonance mode is higher for point $P 1$. Indeed, point $P 1$ corresponds to a maximum (with zero slope for the tangent) for the first elastic mode and corresponds to a zero (with maximum slope for the tangent) for the second elastic mode and then the first resonance is little sensitive to a variation of observation position while the second resonance is very sensitive to a variation of observation position. For the first elastic mode, point $P 2$ corresponds to a larger slope of the tangent than point $P 2$ then the first resonance is more sensitive to a variation of observation position for point $P 2$. As a last remark for Fig. 2 , it can be seen that a stochastic averaging takes place for large frequencies due to the decreasing of the spatial wavelength with respect to frequency.

\subsubsection{Optimal sensor/actuator placement}

It is now assumed that the modal dampings ratio $\xi_{1}$ and $\xi_{2}$ are uncertain and have to be updated using experimental measurements of frequency responses. The experimental frequency response is constructed using the nominal model (with nominal values 0.04 for $\xi_{1}$ and $\xi_{2}$ ) introduced in Section 6.1.1 and the random responses are constructed using the stochastic model introduced in Section 6.1.2. It is assumed that there is no modeling errors and the random variable $\mathbf{N}$ is zero almost surely. For the updating, the frequency responses are restricted to the range $B=[0,433] \times 2 \pi \mathrm{rad} / \mathrm{s}$. The output vector $z$ is made up of the concatenation of the functions $\omega \mapsto \log _{10}\left(\omega\left|\mathbb{U}^{\operatorname{sens}, \mathrm{x}_{\mathrm{s}}}(\omega)\right|\right)$ 
calculated at 10 frequencies regularly spaced in the range $B$. Therefore $n_{z}=10$. The objective is to determine the optimal locations $x_{s}^{\mathrm{opt}}$ and $x_{a}^{\mathrm{opt}}$ for the sensor and the actuator respectively. The prior distributions for the random modal dampings $\Xi_{1}^{\text {prior }}$ and $\Xi_{2}^{\text {prior }}$ are uniform distributions on the interval $[0.02,0.06]$. Two cases are considered. For the Case 1, the modal dampings $\xi_{1}$ and $\xi_{2}$ are considered separately and then two optimal configurations are determined separately. For the Case 2, the modal dampings $\xi_{1}$ and $\xi_{2}$ are considered in a single step and a unique optimal configuration is determined.

For Case 1 , the $\mathrm{KL}$ divergences $\mathcal{K} \mathcal{L}\left(p_{\Xi_{1}^{\text {post }}}, p_{\Xi_{1}^{\text {prior }}} ; x_{s}, x_{a}\right)$ and $\mathcal{K} \mathcal{L}\left(p_{\Xi_{2}^{\text {post }}}\right.$, $\left.p_{\Xi_{2}^{\text {prior }}} ; x_{s}, x_{a}\right)$ are estimated using the Monte Carlo simulation method with $n_{\text {sim }, h}=1000$ realizations. For each realization, the likelihood is estimated using $n_{\text {sim, } p}=800$ realizations of the random positions (as explained in the body of this paper, these 800 realizations are performed at reduced-order level and are therefore not time consuming). The results are shown on Figs. 4 and 5 respectively It can be seen on Fig. 4 that for the updating of modal damping

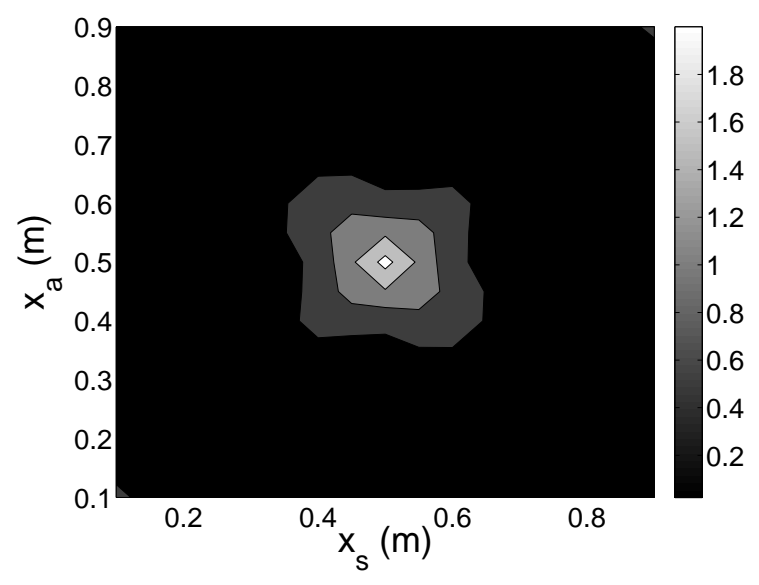

Figure 4: KL divergence $\left(x_{s}, x_{a}\right) \mapsto \mathcal{K} \mathcal{L}\left(p_{\Xi_{1}^{\text {post }}}, p_{\Xi_{1}^{\text {prior }}} ; x_{s}, x_{a}\right)$.

$\xi_{1}$ the optimal configuration is $x_{s}^{o p t}=0.5$ and $x_{a}^{o p t}=0.5$. Indeed, even if all the points of the bar are equally sensitive to $\xi_{1}$, the middle of the bar is, as explained above, the location where the response (for the first resonance) is the most robust with respect to positions $x_{s}$ and $x_{a}$ and is therefore the optimal position for both $x_{s}$ and $x_{a}$. The symmetry with respect to the line $x_{s}=x_{a}$ is due to the reciprocity property of the nominal model and to the 


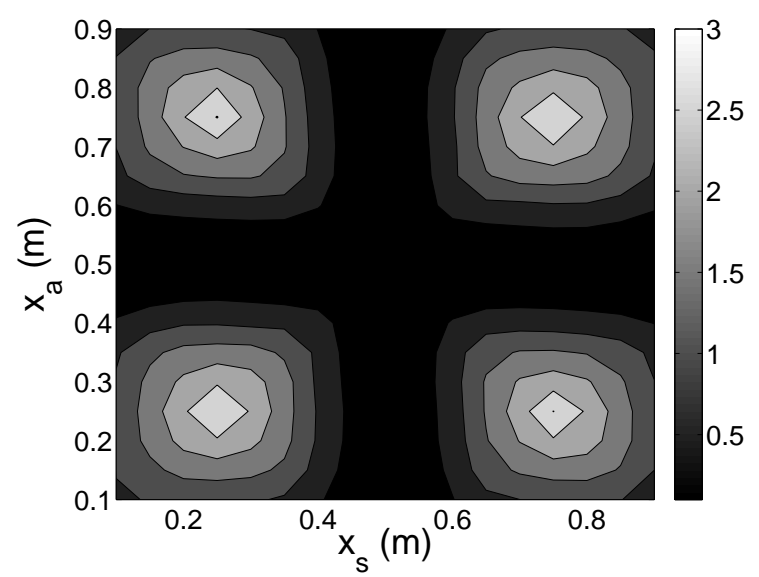

Figure 5: KL divergence $\left(x_{s}, x_{a}\right) \mapsto \mathcal{K} \mathcal{L}\left(p_{\Xi_{2}^{\text {post }}}, p_{\Xi_{2}^{\text {prior }}} ; x_{s}, x_{a}\right)$.

probabilistic models for $X_{s}$ and $X_{a}$ which are identical here. The symmetry with respect to the line $x_{s}+x_{a}=1$ is due to the symmetry of the application with respect to the middle of the bar. Concerning $\xi_{2}$, it can be seen on Fig. 5 that there are four optimal configurations which are such that $x_{s}^{o p t}=0.25$ or $x_{s}^{o p t}=0.75$ and $x_{a}^{o p t}=0.25$ or $x_{a}^{\text {opt }}=0.75$. These configurations correspond to points where the response (for the second resonance) is the most robust with respect to positions $x_{s}$ and $x_{a}$.

For Case 2, the modal dampings $\xi_{1}$ and $\xi_{2}$ are considered in a single step. Let be $\boldsymbol{\xi}=\left[\xi_{1}, \xi_{2}\right]$ and $\boldsymbol{\Xi}=\left[\Xi_{1}, \Xi_{2}\right]$. The KL divergence $\mathcal{K} \mathcal{L}\left(p_{\boldsymbol{\Xi}^{\text {post }}}, p_{\boldsymbol{\Xi}^{\text {prior }}} ; x_{s}, x_{a}\right)$ is plotted on Fig. 6. It can be seen on Fig. 6 that the optimal configurations are again $x_{s}^{\text {opt }}=0.25$ or $x_{s}^{\text {opt }}=0.75$ and $x_{a}^{\text {opt }}=0.25$ or $x_{a}^{\text {opt }}=0.75$. The KL divergence is quite large for the configuration $x_{s}=0.5$ and $x_{a}=0.5$. Indeed this configuration provides very robust information for $\xi_{1}$ and due to uncertainties this configuration also provides few information for $\xi_{2}$.

\subsubsection{Identification of the modal dampings}

The posterior distribution $p_{\boldsymbol{\Xi}^{\text {post }}}\left(\boldsymbol{\xi} \mid \boldsymbol{z}^{\exp }\right)$ is calculated for the optimal configuration $x_{s}^{\text {opt }}=0.25$ and $x_{a}^{\text {opt }}=0.25$ and is plotted on Fig. 7. The marginal posterior distributions $p_{\Xi_{1}^{\text {post }}}\left(\xi_{1} \mid \boldsymbol{z}^{\exp }\right)$ and $p_{\Xi_{2}^{\text {post }}}\left(\xi_{2} \mid \boldsymbol{z}^{\exp }\right)$ are plotted on Fig. 8. It is recalled that the prior distributions for $\Xi_{1}$ and $\Xi_{2}$ are uniform on the interval $[0.02,0.06]$. The estimated mean values are $E\left\{\Xi_{1}\right\}=0.041$ and $E\left\{\Xi_{2}\right\}=0.040$ which are closed from the values from 


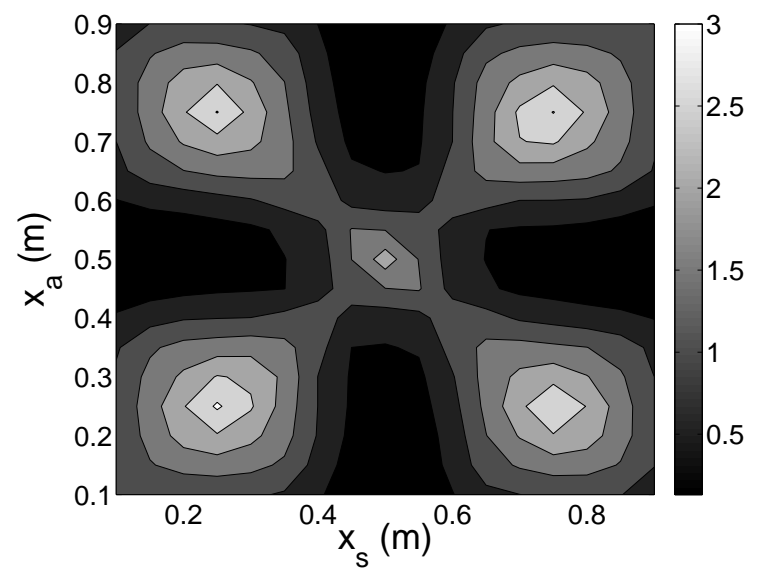

Figure 6: KL divergence $\left(x_{s}, x_{a}\right) \mapsto \mathcal{K} \mathcal{L}\left(p_{\boldsymbol{\Xi}^{\text {post }}}, p_{\boldsymbol{\Xi}^{\text {prior }}} ; x_{s}, x_{a}\right)$.
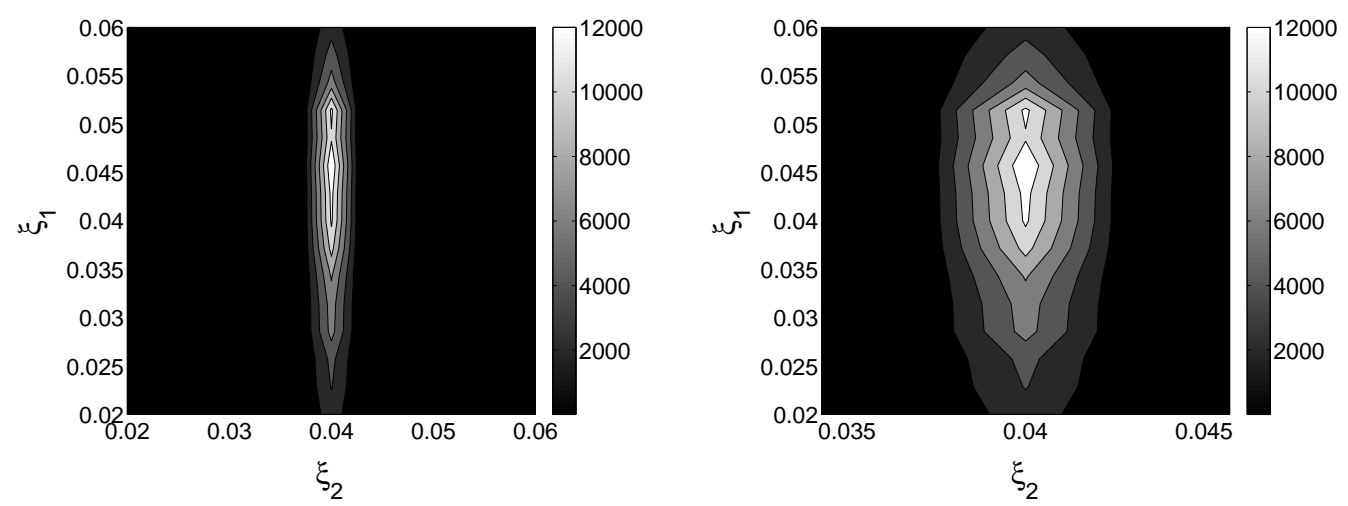

Figure 7: Configuration $x_{s}^{o p t}=0.25$ and $x_{a}^{o p t}=0.25$. Left figure: Posterior distribution $p_{\boldsymbol{\Xi}^{\text {post }}}\left(\boldsymbol{\xi} \mid \boldsymbol{z}^{\mathrm{exp}}\right)$. Right figure: Zoom.

which experimental responses have been generated (0.04). The estimated coefficients of variation (ratio between the standard deviation and the mean value) are $\delta_{\Xi_{1}}=0.24$ and $\delta_{\Xi_{2}}=1.2 \times 10^{-3}$. Then the use of this optimal experimental configuration provides a very robust estimate for the modal damping $\xi_{2}$ and a quite robust estimate for the modal damping $\xi_{1}$, or in other words, the large position uncertainties for the sensor and actuator yields very few errors on the estimation of $\xi_{2}$ and acceptable errors for the estimation of $\xi_{1}$. In order to obtain a very robust estimate for $\xi_{1}$, we can add another 

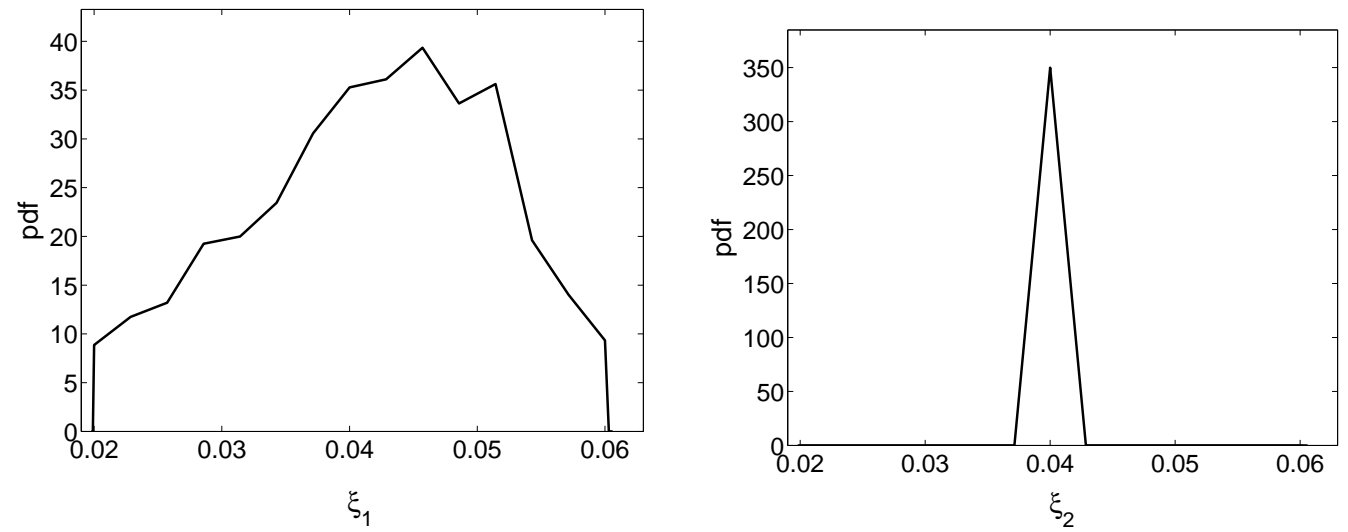

Figure 8: Configuration $x_{s}^{\text {opt }}=0.25$ and $x_{a}^{o p t}=0.25$. Left figure: Marginal posterior distribution $p_{\Xi_{1}^{\text {post }}}\left(\xi_{1} \mid \boldsymbol{z}^{\exp }\right)$. Right figure: Marginal posterior distribution $p_{\Xi_{2}^{\text {post }}}\left(\xi_{2} \mid\right.$ $\left.z^{\exp }\right)$.

sensor or use two experimental configurations. For the configuration $x_{s}=0.5$ and $x_{a}=0.5$, the posterior distribution $p_{\boldsymbol{\Xi}^{\text {post }}}\left(\boldsymbol{\xi} \mid \boldsymbol{z}^{\exp }\right)$ is plotted on Fig. 9 and the marginal posterior distributions $p_{\Xi_{1}^{\text {post }}}\left(\xi_{1} \mid \boldsymbol{z}^{\exp }\right)$ and $p_{\Xi_{2}^{\text {post }}}\left(\xi_{2} \mid \boldsymbol{z}^{\exp }\right)$ are plotted on Fig. 10. It can be seen in these figures that this configuration
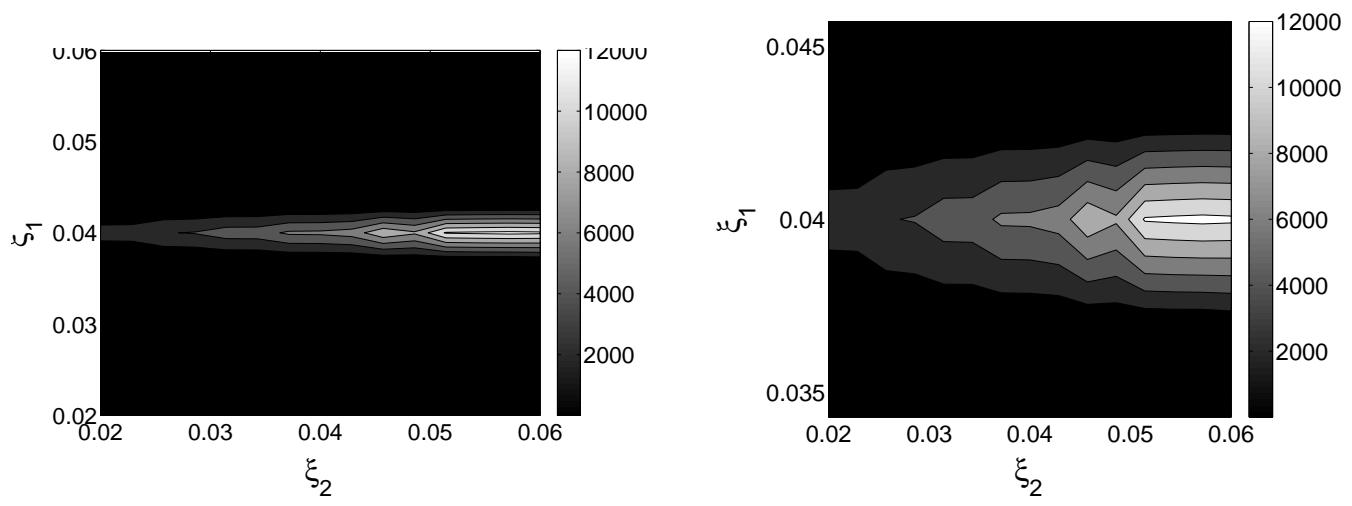

Figure 9: Configuration $x_{s}^{o p t}=0.5$ and $x_{a}^{o p t}=0.5$. Left figure: Posterior distribution $p_{\Xi^{\text {post }}}\left(\boldsymbol{\xi} \mid \boldsymbol{z}^{\exp }\right)$. Right figure: Zoom.

would yield a very robust estimate for $\xi_{1}$ but a poor estimate for $\xi_{2}$. 

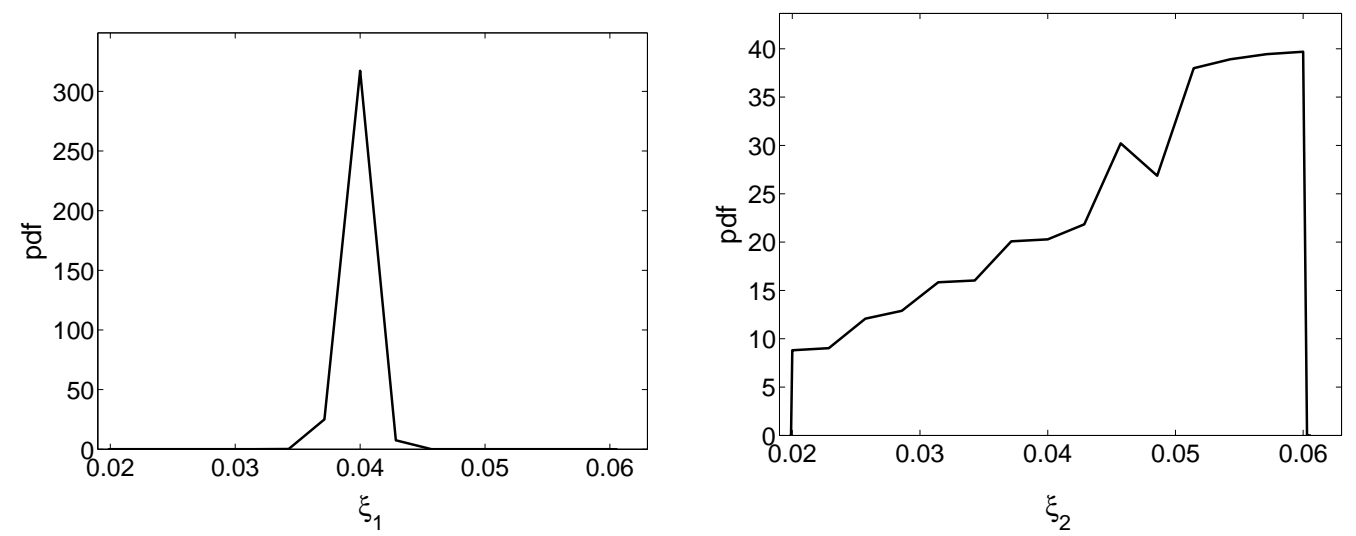

Figure 10: Configuration $x_{s}^{o p t}=0.5$ and $x_{a}^{o p t}=0.5$. Left figure: Marginal posterior distribution $p_{\Xi_{1}^{\text {post }}}\left(\xi_{1} \mid \boldsymbol{z}^{\exp }\right)$. Right figure: Marginal posterior distribution $p_{\Xi_{2}^{\text {post }}}\left(\xi_{2} \mid\right.$ $\left.z^{\exp }\right)$.

\subsection{3-D application}

\subsubsection{Nominal model and its deterministic response}

This second application is related to the plane structure represented in Fig. 11. This structure is made up of two parts: a rigid frame (red plate on Fig. 11) and flexible substructures (blue plates on Fig. 11). The rigid frame has Young's Modulus $2.1 \times 10^{12} \mathrm{~Pa}$, mass density $9800 \mathrm{~kg} / \mathrm{m}^{3}$, Poisson ratio 0.29 and thickness $4.0 \times 10^{-3} \mathrm{~m}$. The flexible plates are all identical and have Young's Modulus $1.7 \times 10^{12} \mathrm{~Pa}$, mass density $7800 \mathrm{~kg} / \mathrm{m}^{3}$, Poisson ratio 0.29 and thickness $4.0 \times 10^{-4} \mathrm{~m}$. The frequency band of analysis is $B=[0 ; 2500] \mathrm{Hz}$. In the band $B$, the structure has 89 elastic modes. There are both global elastic modes which involve displacements of all the structure and local elastic modes for which the displacement is localized on one flexible plate and which are associated with small spatial wavelengths. The damping is modeled by a Rayleigh model so that the damping ratio is 0.04 at frequencies $50 \mathrm{~Hz}$ and $2500 \mathrm{~Hz}$. A unit force is applied at point $P_{1}$ located on the rigid frame (see Fig. 11). The frequency responses of the nominal model are calculated at points $P_{1}, P_{2}$ (located on the rigid frame, see Fig. 11) and $P_{3}$ (located on a flexible plate, see Fig. 11) following the normal direction. The functions $\omega \mapsto \log _{10}\left(\omega\left|\mathbb{U}^{\text {sens, } \mathrm{P}_{1}}(\omega)\right|\right), \omega \mapsto \log _{10}\left(\omega\left|\mathbb{U}^{\text {sens, } \mathrm{P}_{2}}(\omega)\right|\right)$ and $\omega \mapsto \log _{10}\left(\omega\left|\mathbb{U}^{\text {sens, } \mathrm{P}_{3}}(\omega)\right|\right)$, in which $\mathbb{U}^{\text {sens, } \mathrm{P}_{\mathrm{i}}}(\omega)$ is the displacement of point $P_{i}$ following the normal direction, are plotted on Figs. 12, 13 and 14. 


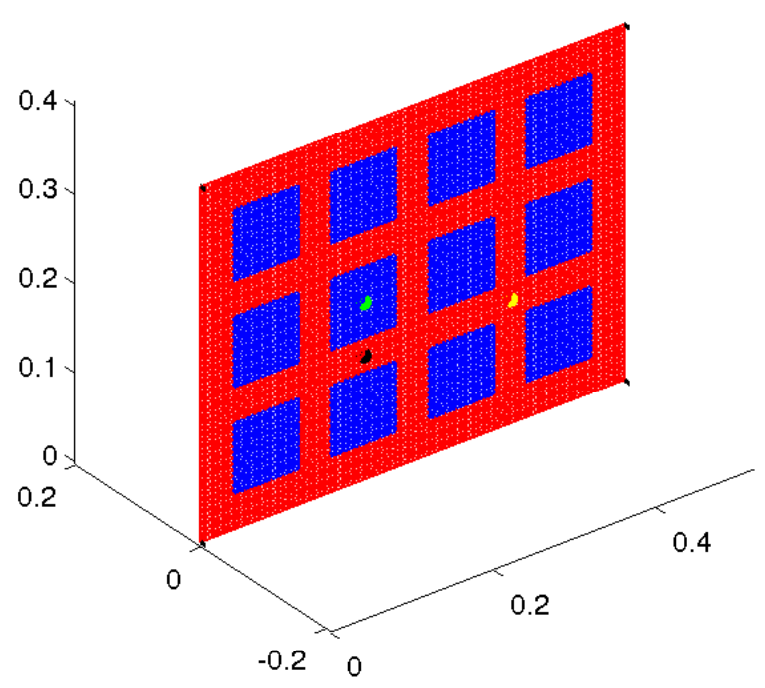

Figure 11: FE mesh of the structure. Point $P_{1}$ (black), point $P_{2}$ (yellow) and point $P_{3}$ (red).

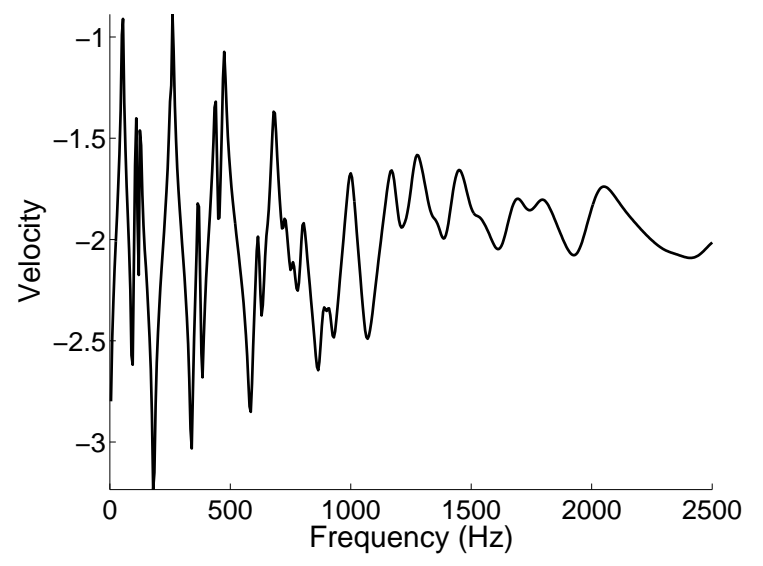

Figure 12: Frequency response in velocity for point $P_{1}$.

6.2.2. Stochastic model of positioning uncertainties and stochastic response

Concerning the applied force, the fluctuations of the position around the nominal value are modelled by a random vector for which the probability 


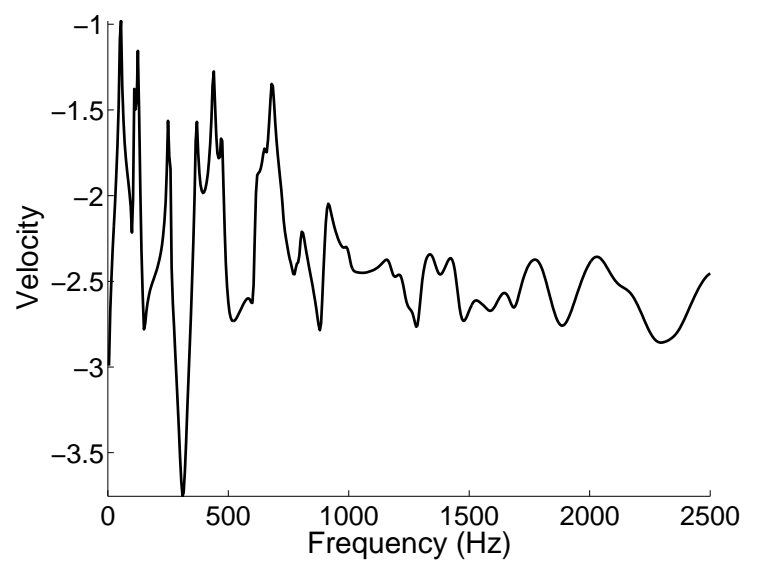

Figure 13: Frequency response in velocity for point $P_{2}$.

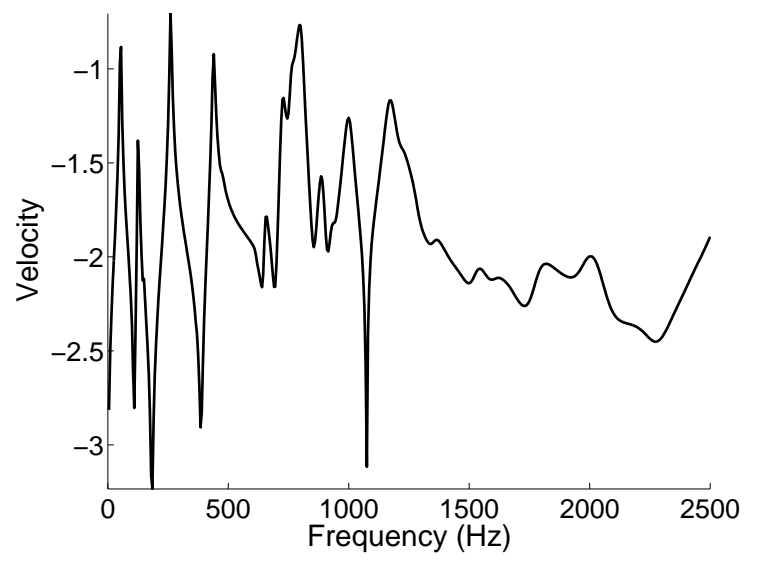

Figure 14: Frequency response in velocity for point $P_{3}$.

distribution is uniform on a disc of diameter $0.015 \mathrm{~m}$ and the random angles of orientation following the three directions are independent uniform random variables on the support $[-0.21 ; 0.21]$ rad. Concerning the observed response, the fluctuations of the position around the nominal value are modelled by a random vector for which the probability distribution is uniform on a disc of diameter $0.01 \mathrm{~m}$ and the random angles of orientation following the three directions are independent uniform random variables on the support $[-0.21 ; 0.21] \mathrm{rad}$. For points $P_{1}, P_{2}$ and $P_{3}$, the random frequency 
responses are calculated using the Monte Carlo simulation method with 1000 realizations. Several cases of randomness are analysed. For Case 1, only the positions are random while the rotations are kept zero. The corresponding random frequency responses are plotted on Figs. 15, 16 and 17. For point $P_{1}$, the convergence of the L2-norm of the mean value with respect to the number of Monte Carlo simulations is plotted on Fig. 18. It can be seen

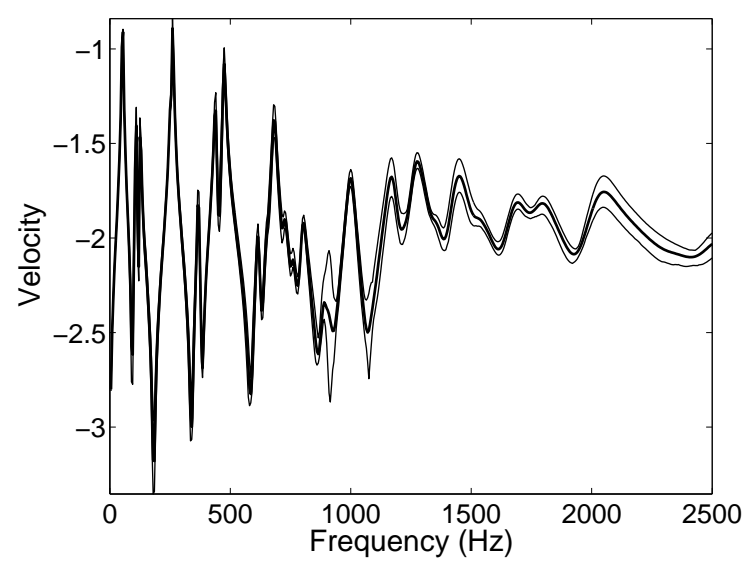

Figure 15: Case 1: Random frequency response in velocity for point $P_{1}$ : Mean value (thick line) and confidence region with probability level 0.95 (thin lines).

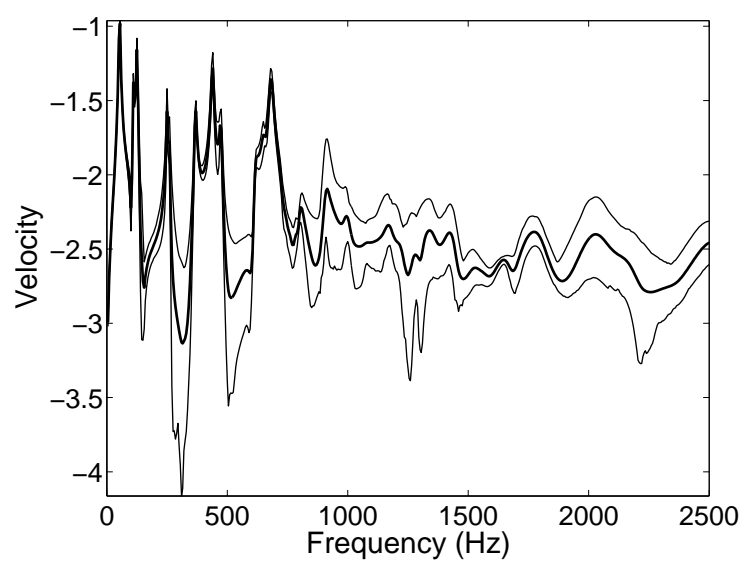

Figure 16: Case 1: Random frequency response in velocity for point $P_{2}$ : Mean value (thick line) and confidence region with probability level 0.95 (thin lines). 


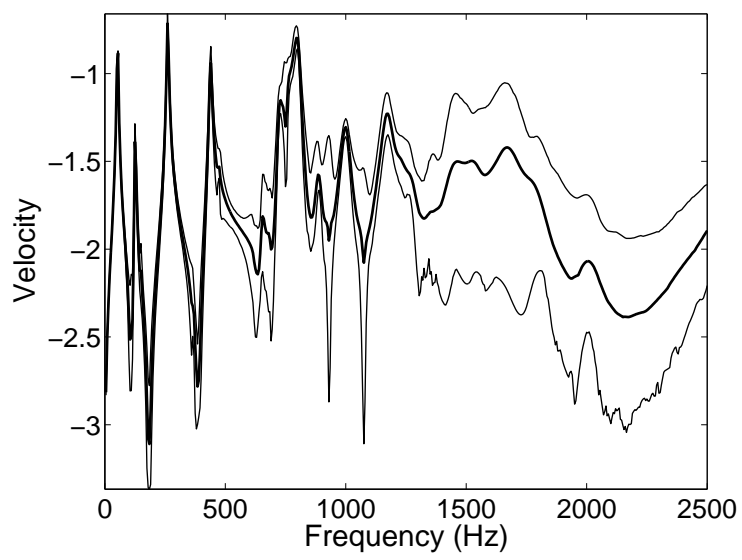

Figure 17: Case 1: Random frequency response in velocity for point $P_{3}$ : Mean value (thick line) and confidence region with probability level 0.95 (thin lines).

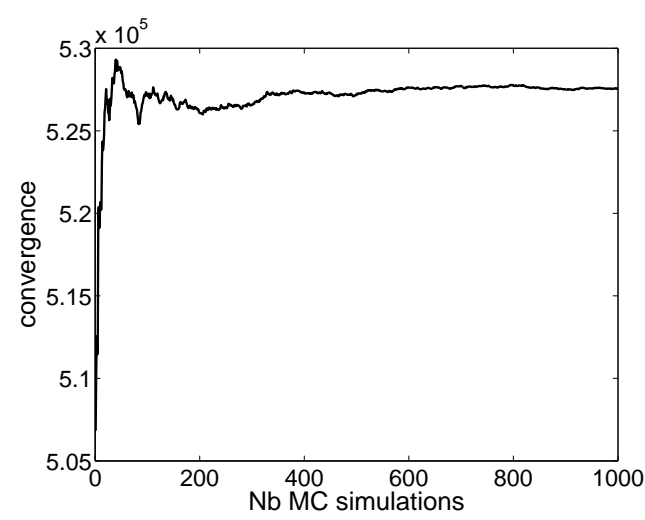

Figure 18: Convergence of the mean value with respect to the number of Monte Carlo simulations.

that the fluctuations of the response increase with frequency since the spatial wavelengths decrease with frequency. Concerning point $P_{3}$ located on a flexible plate, the fluctuations become very large since small-size spatial wavelength modes are involved. For Case 2, the positions are deterministic while the rotations are random. The corresponding random frequency responses are plotted on Figs. 19, 20 and 21. It can be seen on these figures that, compared to the previous case, the fluctuations of the response are more homogeneous with respect to frequency. Indeed, the randomness of 


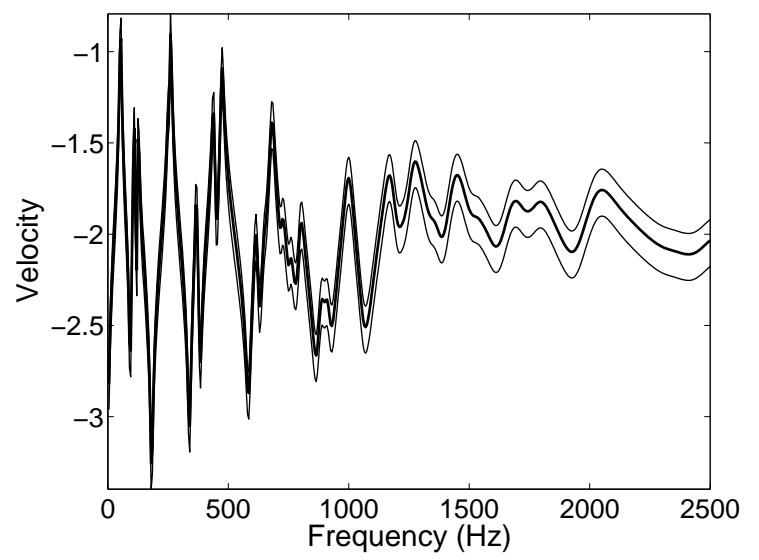

Figure 19: Case 2: Random frequency response in velocity for point $P_{1}$ : Mean value (thick line) and confidence region with probability level 0.95 (thin lines).

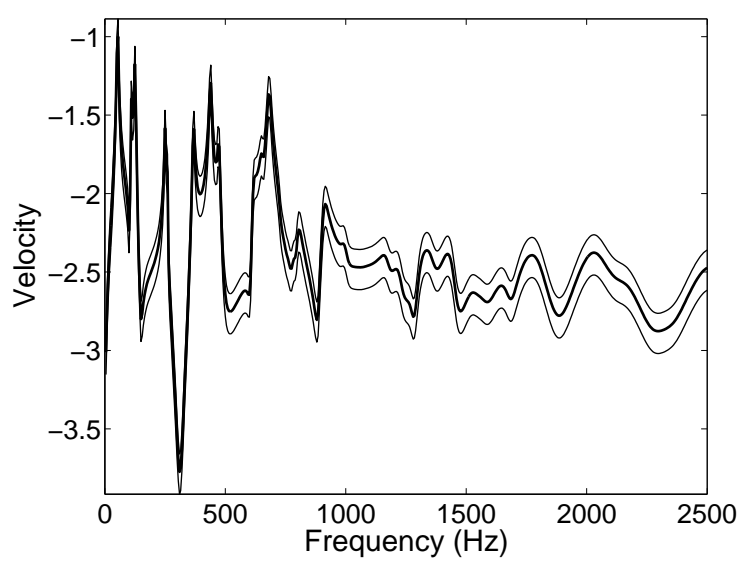

Figure 20: Case 2: Random frequency response in velocity for point $P_{2}$ : Mean value (thick line) and confidence region with probability level 0.95 (thin lines).

the rotation yields randomness on the response independently of the spatial wavelength. For Case 3, the positions and the rotations are both random. The corresponding random frequency responses are plotted on Figs. 22, 23 and 24. For this case, the two sources of randomness are cumulated yielding large increasing fluctuations in band $B$. 


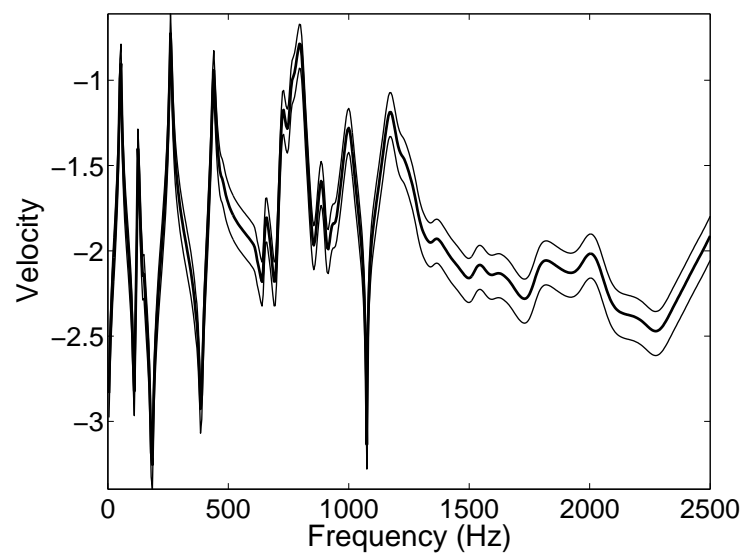

Figure 21: Case 2: Random frequency response in velocity for point $P_{3}$ : Mean value (thick line) and confidence region with probability level 0.95 (thin lines).

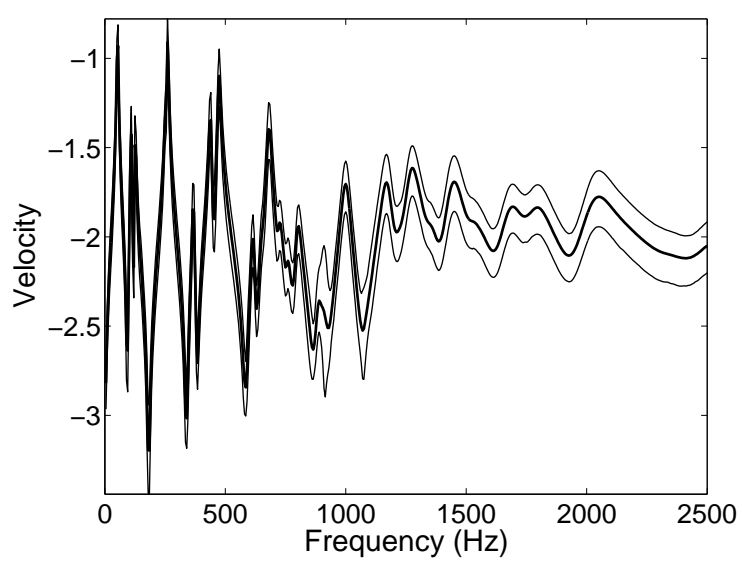

Figure 22: Case 3: Random frequency response in velocity for point $P_{1}$ : Mean value (thick line) and confidence region with probability level 0.95 (thin lines).

\subsubsection{Identification of the Young's moduli}

The Young's modulus $e_{1}$ of the flexible plates and the Young's modulus $e_{2}$ of the rigid frame are now supposed to be unknown and have to be identified using experimental frequency responses and the random frequency responses calculated using the stochastic computational model at 20 frequencies regularly spaced in the interval $B$. For each point $P_{i}, i \in\{1,2,3\}$, the output vector $\boldsymbol{z}$ is made up of the the concatenation of the functions 


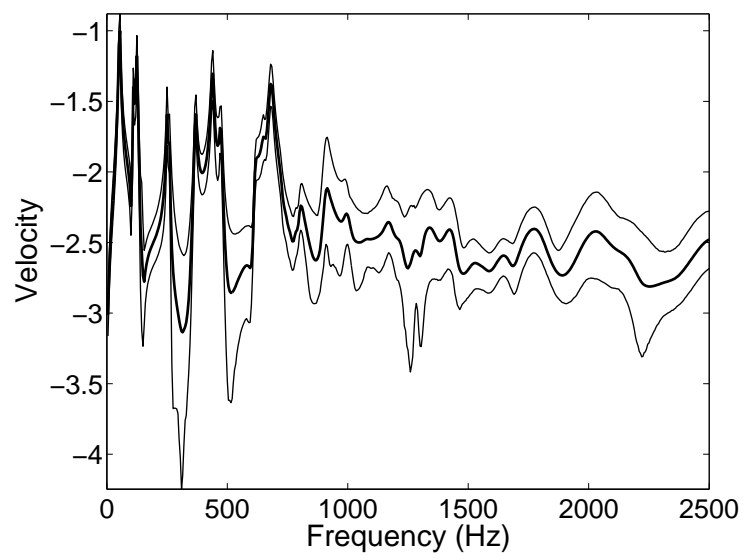

Figure 23: Case 3: Random frequency response in velocity for point $P_{2}$ : Mean value (thick line) and confidence region with probability level 0.95 (thin lines).

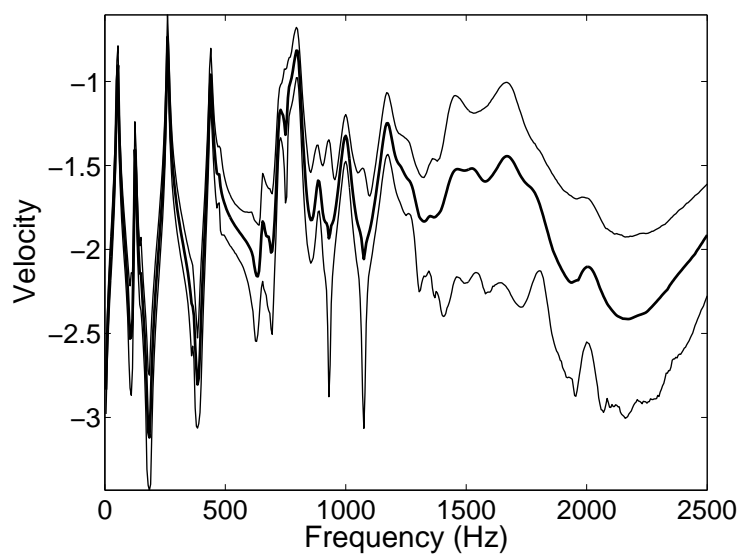

Figure 24: Case 3: Random frequency response in velocity for point $P_{3}$ : Mean value (thick line) and confidence region with probability level 0.95 (thin lines).

$\omega \mapsto \log _{10}\left(\omega\left|\mathbb{U}^{\text {sens, }, P_{i}}(\omega)\right|\right)$ calculated at the 20 frequencies of analysis. Therefore $n_{z}=20$. Let $E_{1}$ be the random Young's modulus of the flexible plates, let $E_{2}$ be the random Young's modulus of the rigid frame and let be $\boldsymbol{e}=\left(e_{1}, e_{2}\right)$ and $\boldsymbol{E}=\left(E_{1}, E_{2}\right)$. The prior probability distribution are uniform distributions on the intervals $\left[2.05 \times 10^{12}, 2.15 \times 10^{12}\right] \mathrm{Pa}$ for $E_{1}$ and $\left[1.65 \times 10^{12}, 1.75 \times 10^{12}\right] \mathrm{Pa}$ for $E_{2}$. The identification is performed using only one observation point that has to be chosen beyond $P_{1}, P_{2}$ and $P_{3}$. To 
make the choice, the KL divergence is estimated for each point. The KL divergence $\mathcal{K} \mathcal{L}\left(p_{\boldsymbol{E}^{\text {post }}}, p_{\boldsymbol{E}^{\text {prior }}}\right)$ is estimated using the Monte Carlo simulation method with $n_{\text {sim, }}=800$ realizations. For each realization, the likelihood is estimated using $n_{s i m, p}=800$ realizations of the random position/orientation. For point $P_{1}$ the KL divergence is 2.48 , for point $P_{2}$ the KL divergence is 1.63 and for point $P_{3}$ the KL divergence is 2.17 . As a conclusion, the most informative observation point is $P_{1}$. This result confirms the low sensitivity of the frequency response at $P_{1}$ with respect to position/orientation uncertainties (see Fig. 22). One of the realization of the frequency response calculated in Section 6.2.3 is chosen as the experimental response. This experimental response is plotted on Fig. 25. For point $P_{1}$, the posterior distribution of

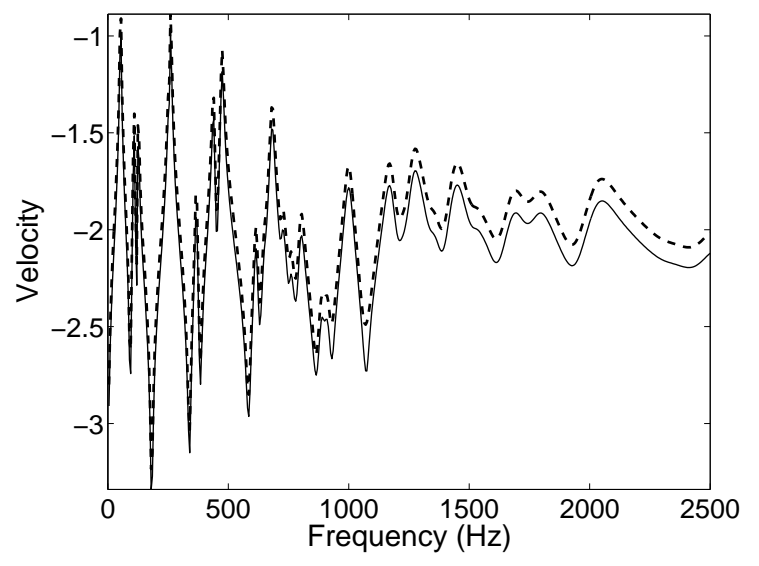

Figure 25: Experimental response (thick dashed line) and response of the nominal model (thin solid line).

random variable $\boldsymbol{E}$ is plotted on Fig. 26. The marginal posterior distribution of random variables $E_{1}$ and $E_{2}$ are plotted on Fig. 27. The estimated mean values are $1.7 \times 10^{12} \mathrm{~Pa}$ for $E_{1}$ and $2.1 \times 10^{12} \mathrm{~Pa}$ for $E_{2}$. The estimated coefficients of variation are $1.4 \times 10^{-3}$ for $E_{1}$ and $1.1 \times 10^{-3}$ for $E_{2}$. It can then be concluded that the choice of $P_{1}$ yields a robust estimation for the two Young's moduli. The posterior distributions have also been calculated for point $P_{3}$ and are plotted on Figs. 28 and 29. The corresponding estimated mean values are $1.7 \times 10^{12} \mathrm{~Pa}$ for $E_{1}$ and $2.1 \times 10^{12} \mathrm{~Pa}$ for $E_{2}$. The corresponding estimated coefficients of variation are $2.6 \times 10^{-3}$ for $E_{1}$ and $2.1 \times 10^{-3}$ for $E_{2}$. It can be concluded from Figs. 28 and 29 , that as expected, the choice of $P_{3}$ yields a poor estimation for the Young's modulus of the flex- 

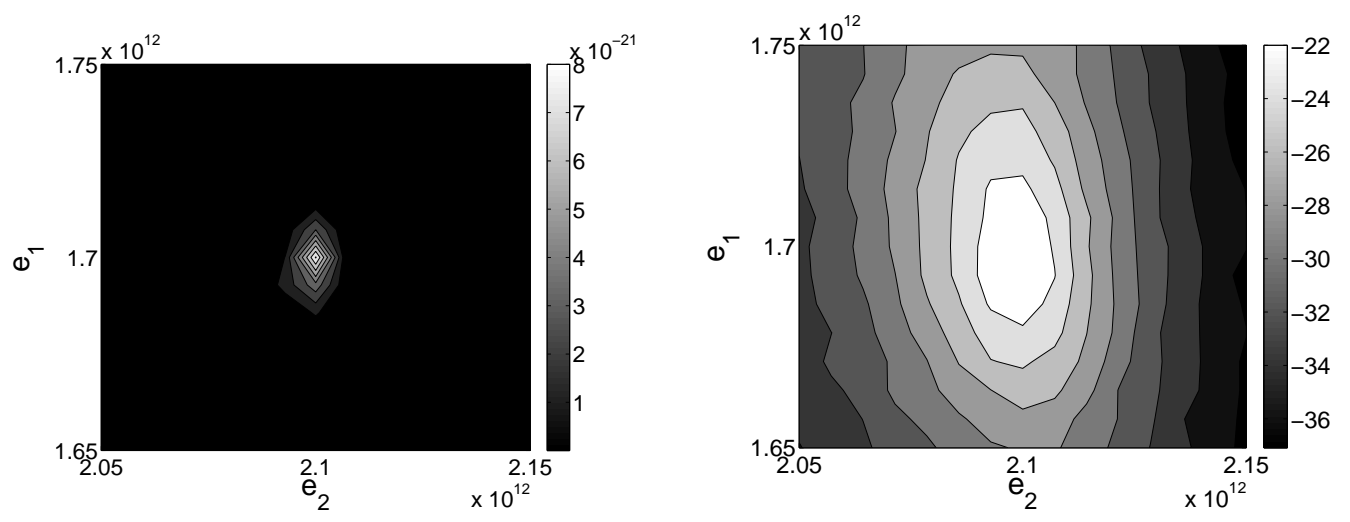

Figure 26: Observation at point $P_{1}$. Left figure: Posterior distribution $\boldsymbol{e} \mapsto p_{\boldsymbol{E}^{\text {post }}}(\boldsymbol{e}$ $\left.\boldsymbol{z}^{\exp }\right)$. Right figure: function $\boldsymbol{e} \mapsto \log _{10}\left(p_{\boldsymbol{E}^{\text {post }}}\left(\boldsymbol{e} \mid \boldsymbol{z}^{\exp }\right)\right)$.
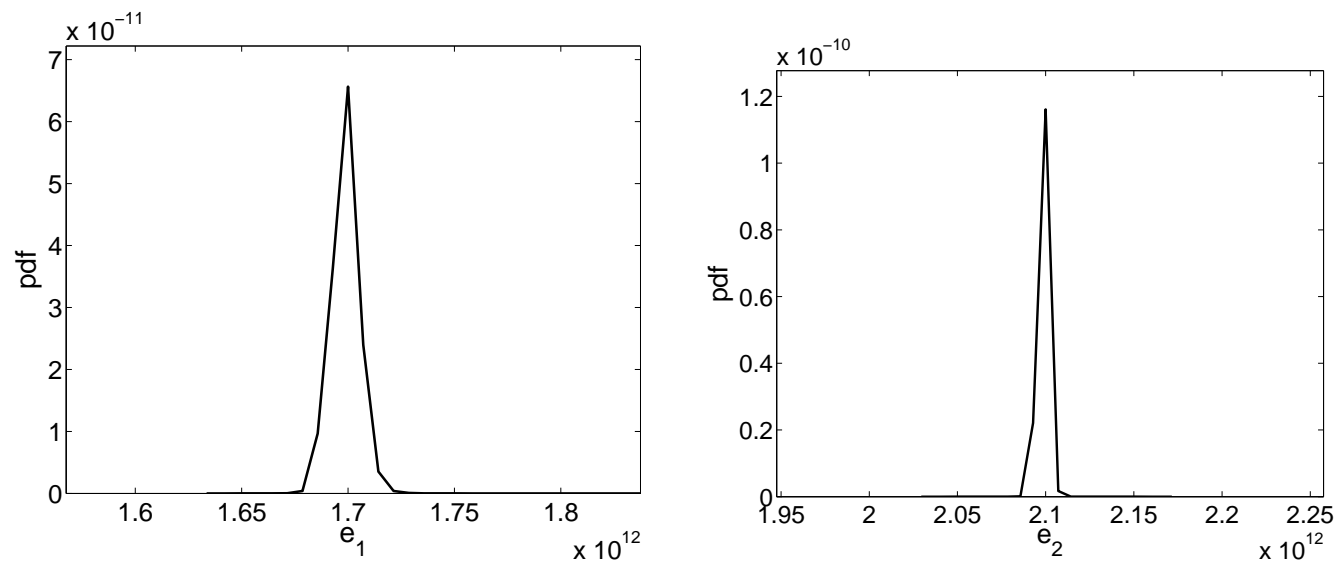

Figure 27: Observation at point $P_{1}$. Left figure: Marginal posterior distribution $p_{E_{1}^{\text {post }}}\left(e_{1} \mid\right.$ $\left.\boldsymbol{z}^{\exp }\right)$. Right figure: Marginal posterior distribution $p_{E_{2}^{\text {post }}}\left(e_{2} \mid \boldsymbol{z}^{\exp }\right)$.

ible plates. This result is due to the small size of the spatial wavelength of the elastic modes that contributes to the response at point $P_{3}$ located on a flexible plate. 

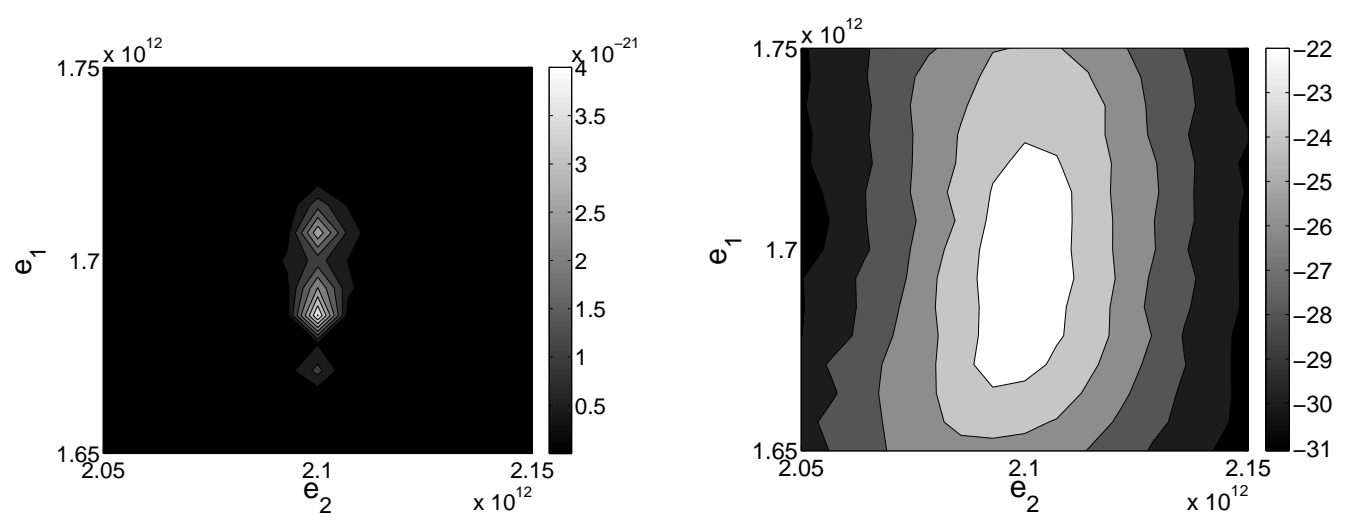

Figure 28: Observation at point $P_{3}$. Left figure: Posterior distribution $\boldsymbol{e} \mapsto p_{\boldsymbol{E}^{\text {post }}}(\boldsymbol{e}$ $\left.\boldsymbol{z}^{\exp }\right)$. Right figure: function $\boldsymbol{e} \mapsto \log _{10}\left(p_{\boldsymbol{E}^{\text {post }}}\left(\boldsymbol{e} \mid \boldsymbol{z}^{\exp }\right)\right)$.
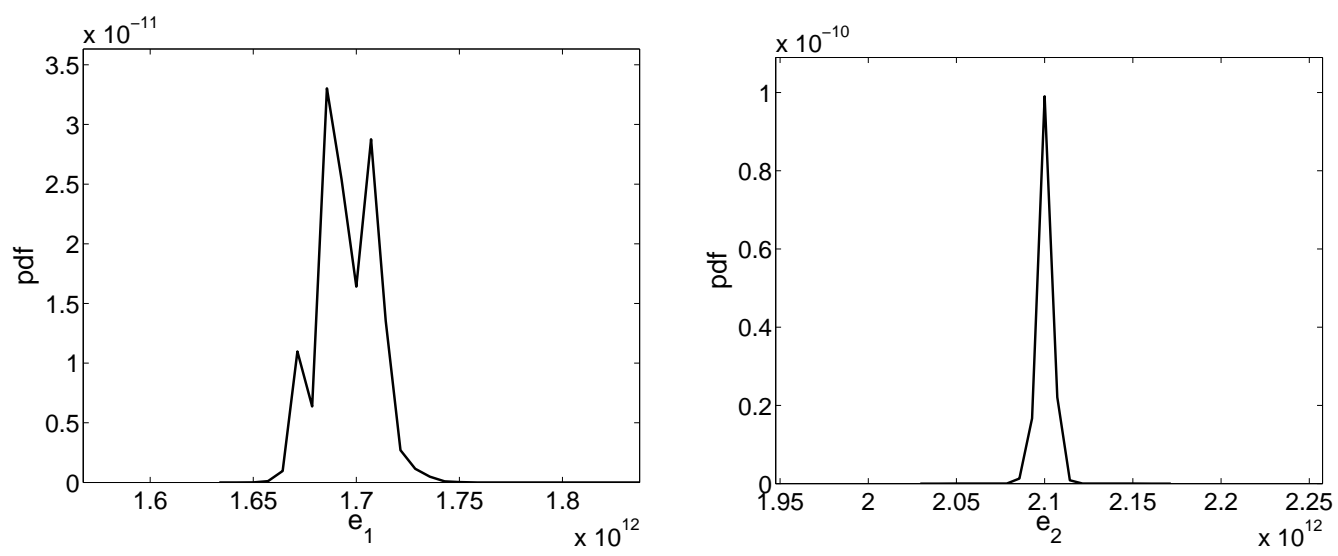

Figure 29: Observation at point $P_{3}$. Left figure: Marginal posterior distribution $p_{E_{1}^{\text {post }}}\left(e_{1} \mid\right.$ $\left.\boldsymbol{z}^{\exp }\right)$. Right figure: Marginal posterior distribution $p_{E_{2}^{\text {post }}}\left(e_{2} \mid \boldsymbol{z}^{\exp }\right)$.

\section{Conclusions}

We have presented a probabilistic method to take into account uncertainties related to the position and orientation of the sensors and actuators. This methodology allows (1) to analyse the propagation of these uncertainties into the dynamical response of the structure, (2) to take into account these uncertainties in the updating of the computational model using a Bayesian method and (3) to find the optimal placement for the sensors and actua- 
tors. This methodology is little intrusive with respect to the commercial softwares and is well adapted to parallel computing. The sensitivity of the dynamical response to sensor/actuator position uncertainties is larger for locations where elastic modes with small-size spatial wavelength have large contributions yielding inaccuracy for updated values of the parameters of the computational model. The methodology we have proposed allows to detect automatically such undesirable locations for the sensors and actuators.

\section{Acknowledgements}

This research work has been carried out in the context of the FUI 20122015 SICODYN Project (pour des SImulations crédibles via la COrrélation calculs-essais et l'estimation des incertitudes en DYNamique des structures). The support of the FUI (Fonds Unique Interministériel) is gratefully acknowledged.

[1] M. Baruch, Modal data are insufficient for identification of both mass and stiffness matrices, AIAA journal, 35(11) (1997) 1797-1798.

[2] J.L. Beck, L.S. Katafygiotis, Updating models and their uncertaintiesBayesian statistical framework, American Society of Civil Engineers, Journal of Engineering Mechanics, 124 (4) (1998), 455-461.

[3] J. Beck, K. Yuen, Model Selection Using Response Measurements: Bayesian Probabilistic Approach, J. Eng. Mech., 130(2) (2004) 192-203.

[4] A. T. Bollinger, Finite element model updating for FEA/EMA modal correlation via constrained optimization theory, IMAC Proc., 882-888, 1994.

[5] R. Castro-Triguero, S. Murugan, R. Gallego, M. I. Friswell, Robustness of optimal sensor placement under parametric uncertainty, Mechanical Systems and Signal Processing, 41(1-2) (2013) 268-287.

[6] K. Chaloner, I. Verdinelli, Bayesian experimental design: a review, Statistical Science 10(3) (1995) 273-304.

[7] S. Cogan, D. Lenoir, G. Lallement, An improved frequency response residual for model correction, IMAC Proc., 568-573, 1995. 
[8] A. Eiben, J. Smith, Introduction to Evolutionary Computing, Springer, 2003.

[9] A. Gelman, J. Carlin, H. Stern, D. Rubin, Bayesian Data Analysis, Second Edition, Chapman \& Hall, London, 2004.

[10] M. I. Friswell, J. E. Mottershead, Finite Element Model Updating in Structural Dynamics, Kluwer Academic Publishers, 1995.

[11] M. I. Friswell, D. J. Inman, D. F. Pilkey, Direct updating of damping and stiffness matrices, AIAA journal, 36(3) (1998), 491-493.

[12] E. T. Jaynes, Information theory and statistical mechanics, Phys. Rev., 106(4) (1957) 620-630 and 108(3) (1957) 171-190.

[13] J. N. Kapur, H. K. Kesavan, Entropy Optimization Principles with Applications, Academic Press, San Diego, 1992.

[14] S. Kullback, R. Leibler, On information and sufficiency, Annals of Mathematical Statistics, 22 (1951) 79-86.

[15] R. Ohayon, C. Soize, Structural Acoustics and Vibration, Academic Press, San Diego, 1998.

[16] C. Papadimitriou, J. L. Beck, S. K. Au, Entropy-Based Optimal Sensor Location for Structural Model Updating, Journal of Vibration and Control, 6, (2000) 781-800.

[17] C. Papadimitriou, Optimal sensor placement methodology for parametric identification of structural systems, Journal of Sound and Vibration, 278(4-5) (2004) 923-947.

[18] C. Papadimitriou, G. Lombaert, The effect of prediction error correlation on optimal sensor placement in structural dynamics, Mechanical Systems and Signal Processing, 28 (2012) 105-127.

[19] T. G. Ritto, Choice of Measurement Locations of Nonlinear Structures Using Proper Orthogonal Modes and Effective Independence Distribution Vector, Shock and Vibration, Article ID 697497 (2014).

[20] R. Y. Rubinstein, D. P. Kroese, Simulation and the Monte Carlo Method, 2nd edn, John Wiley and Sons, New York, 2008. 
[21] M. J. Schultz, D. J. Inman, Model updating using constrained eigenstructure assignment, Journal of Sound and Vibration, 178(1) (1994) 113-130.

[22] C. E. Shannon, A mathematical theory of communication, Bell System Technology Journal, 27 (1948) 379-423 and 623-659.

[23] C. Soize, Stochastic Models of Uncertainties in Computational Mechanics, American Society of Civil Engineers (ASCE), Reston, 2012.

[24] J. C. Spall, Introduction to Stochastic Search and Optimization, John Wiley and Sons, Hoboken, New Jersey, 2003.

[25] E. Walter, L. Pronzato, Identification of Parametric Models from Experimental Data, Springer-Verlag, Berlin, 1997.

[26] A.A. Shabana, Dynamics of Multibody Systems, Cambridge University Press, 2003.

[27] M. P. Wand, M. C. Jones, Kernel Smoothing, Chapman \& Hall, London, U.K., 1995.

[28] N. Xiong, P. Svensson, Multi-sensor management for information fusion: issues and approaches, Information Fusion, 3(2) (2002) 163-186.

[29] D. C. Zimmerman, T. Simmermacher, Model correlation using multiple static load and vibration tests, AIAA journal, 33(11) (1995) 2182-2188. 